\title{
Transverse Momentum and Pseudorapidity Spectrum of the Top Quark, Lepton, and $b$ Jet in Proton-Proton Collisions at LHC
}

\author{
Li-Na Gao $\mathbb{D}$ and Er-Qin Wang $(\mathbb{D}$ \\ Department of Physics, Taiyuan Normal University, Jinzhong, Shanxi 030619, China \\ Correspondence should be addressed to Li-Na Gao; gao-lina@qq.com
}

Received 8 December 2020; Revised 12 January 2021; Accepted 2 April 2021; Published 23 April 2021

Academic Editor: Bhartendu K. Singh

Copyright @ $2021 \mathrm{Li}-\mathrm{Na}$ Gao and Er-Qin Wang. This is an open access article distributed under the Creative Commons Attribution License, which permits unrestricted use, distribution, and reproduction in any medium, provided the original work is properly cited. The publication of this article was funded by SCOAP ${ }^{3}$.

We study the transverse momentum and pseudorapidity spectrum of the top quark and their decay products, the $t \bar{t}$ system, and the total number of jets in proton-proton ( $\mathrm{pp}$ ) collisions at $13 \mathrm{TeV}$ by using the Tsallis-Pareto-type function and the three-source Landau hydrodynamic model, respectively. The related parameters, such as the effective temperature of the interacting system $(T)$, the nonextensivity of the process $(n)$, and the width $(\sigma)$ of pseudorapidity distribution, are extracted.

\section{Introduction}

The top quark is an interesting particle. It was first found by the Tevatron detector of Fermi National Accelerator Laboratory in 1995 [1-3]. As the heaviest particle in the Standard Model, the top quark is very different from the other quark. Its biggest mass is one of the hotspots for physicists. And it is the only one quark which will decay before it becomes hadron. These unique properties make top quark occupy a very important position in particle physics.

The Large Hadron Collider (LHC) has created a lot of top quark events since 2010. Physicists could study the top quark in detail with the help of the LHC. By studying the final state particles produced in high-energy collisions, physicists could obtain some information about the evolution of the collision system. The spectra of transverse momenta $\left(p_{T}\right)$ and (pseudo)rapidity $(\eta / y)$ are important quantities measured in experiments. Many models and functions are used to describe the transverse momentum and pseudorapidity spectra.

In this paper, we use the Tsallis-Pareto-type function [4, 5] and the three-source Landau hydrodynamic model [6] to describe the transverse momentum and pseudorapidity spectra of the top quark, lepton, and bottom quark produced in proton-proton (pp) collisions at the center of mass energy $\sqrt{s}=13 \mathrm{TeV}$ measured at the parton level and particle level, in the full phase space and in the fiducial phase by CMS Collaboration at the LHC [7]. The values of related parameters are extracted and analyzed.

The present paper is organized as follows. We briefly introduce the Tsallis-Pareto-type function and the threesource Landau hydrodynamic model in Section 2. The result of comparisons with experimental data is given in Section 3. Moreover, we outlined the conclusions in Section 4.

\section{The Model and Formalism}

2.1. The Tsallis-Pareto-Type Function. There are some models and functions that could be used to analyze the transverse momentum distribution, such as the Erlang distribution [8-10], the inverse power law [11-14], the Schwinger mechanism $[9,10,15,16]$, Lévy distribution [16], and the Tsallis statistics [17-22]. In a lot of models and formulas, the Tsallis-Pareto-type function is a good choice for transverse momentum spectra. Whether in the relative high $p_{T}$ region or the relative low $p_{T}$ region, people could use the Tsallis-Pareto-type function to fit the experimental data well 


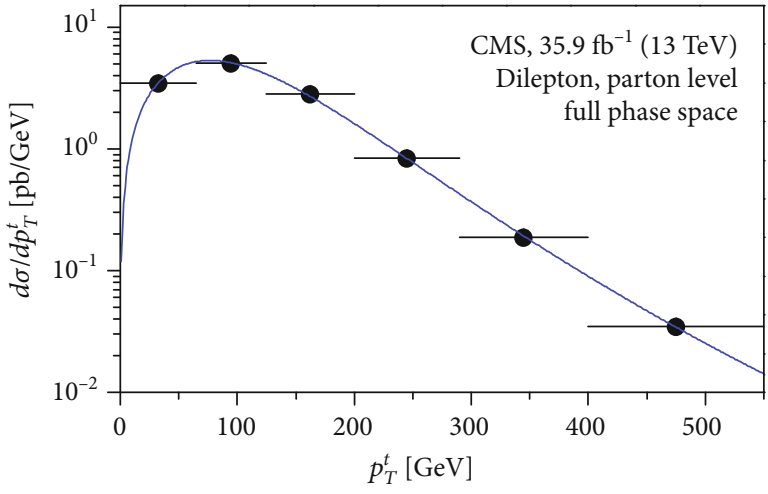

- Data

Tsallis-Pareto-type function

(a)

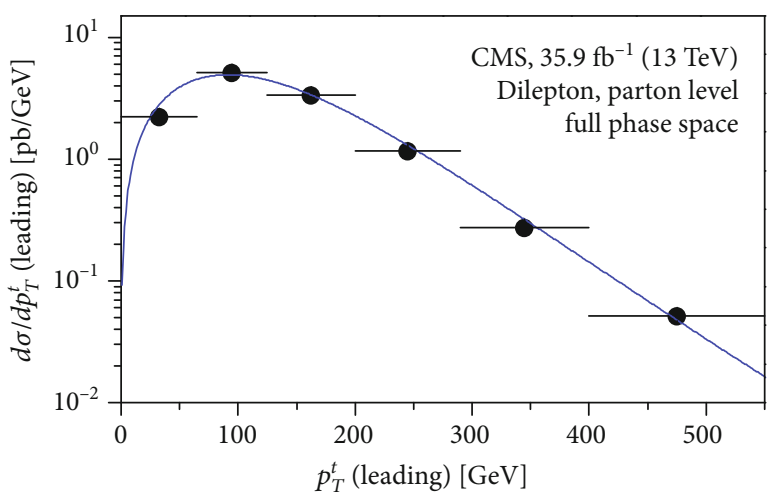

- Data

Tsallis-Pareto-type function

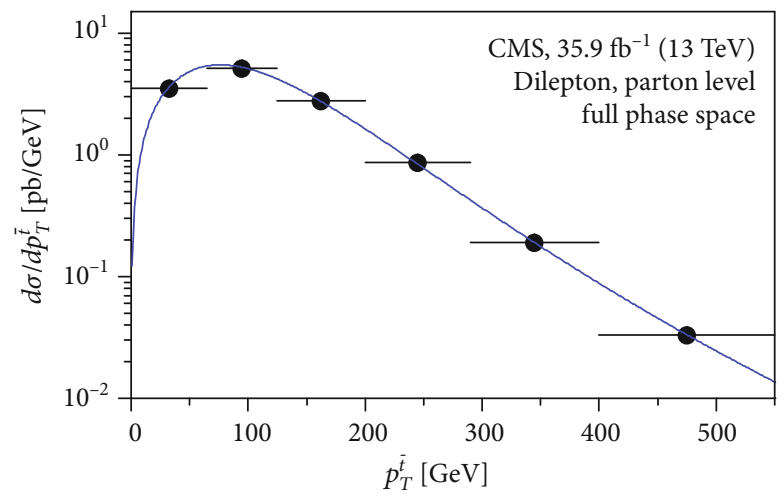

- Data

Tsallis-Pareto-type function

(b)

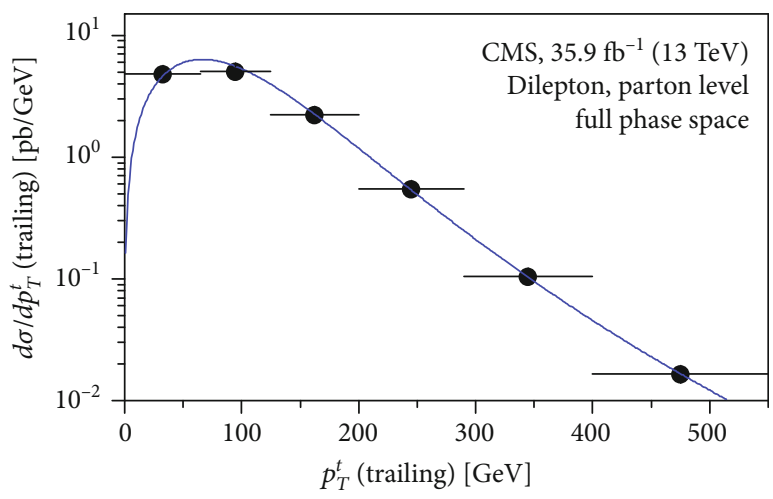

- Data

Tsallis-Pareto-type function

(d)

(c)

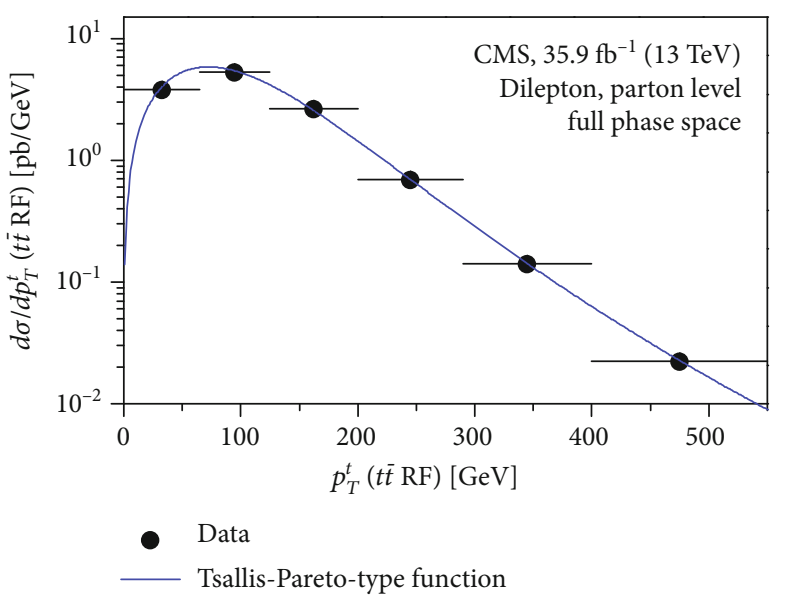

(e)

FIGURE 1: Transverse momentum distribution of (a) the top quark $\left(p_{T}^{t}\right)$, (b) the top antiquark $\left(p_{T}^{\bar{t}}\right)$, (c) the top quark or top antiquark with the largest $p_{T}\left(p_{T}^{t}\right.$ (leading)), (d) the top quark or top antiquark with the second-largest $p_{T}\left(p_{T}^{t}\right.$ (trailing)), and (e) the top quark in the rest frame of the $t \bar{t}$ system $\left(p_{T}^{t}(t \bar{t} \mathrm{RF})\right)$ at the parton level in the full phase space produced in pp collisions at $\sqrt{s}=13 \mathrm{TeV}$. The solid circles represent the experimental data of the CMS Collaboration in literature [7]; the curves are our results calculated by the Tsallis-Pareto-type function. 


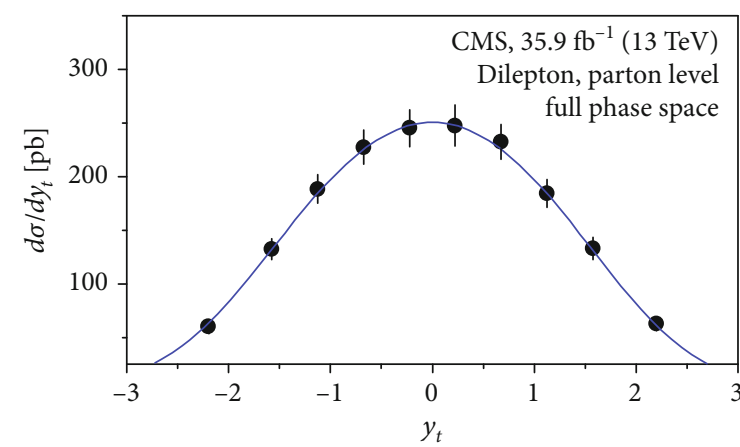

- Data

three-Gaussian function

(a)

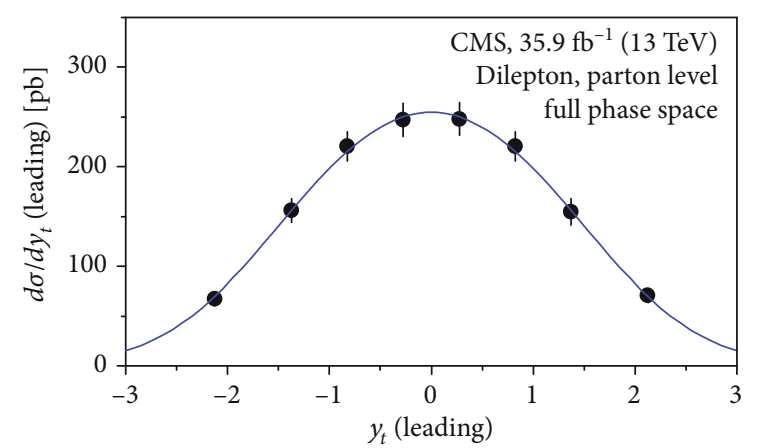

- Data

three-Gaussian function

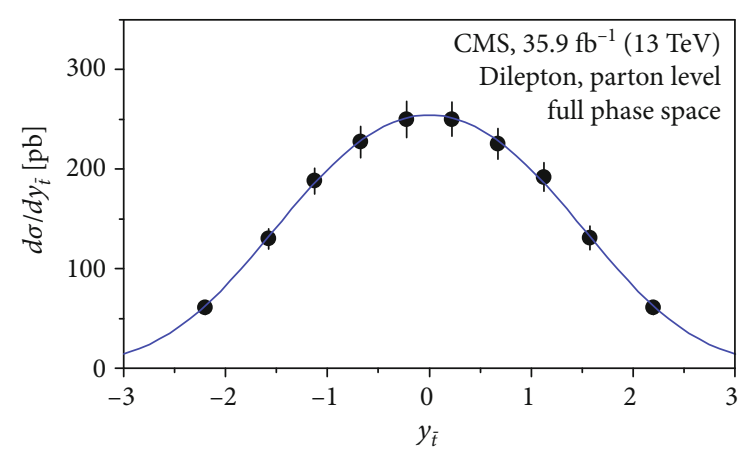

- Data

three-Gaussian function

(b)

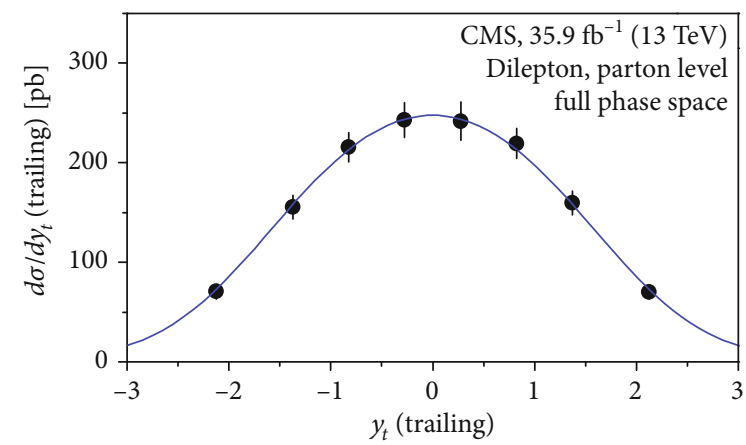

- Data

three-Gaussian function

(c)

(d)

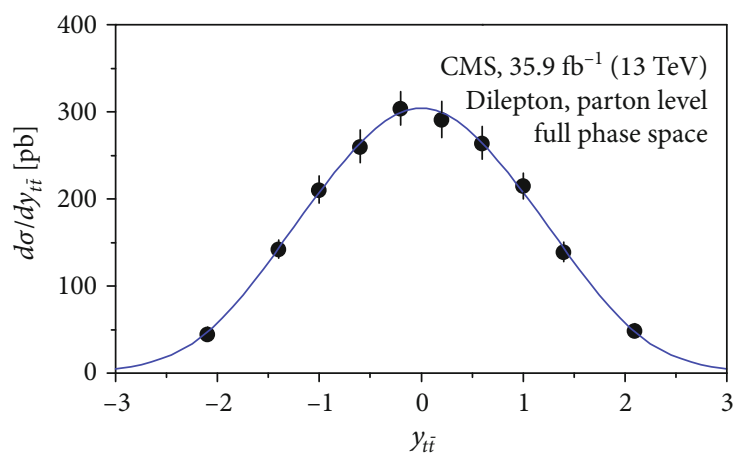

- Data

three-Gaussian function

(e)

FiguRE 2: Rapidity distributions of (a) the top quark $\left(y_{t}\right),(\mathrm{b})$ the top antiquark $\left(y_{\bar{t}}\right)$, (c) the top quark or top antiquark with the largest $p_{T}\left(y_{t}\right.$ (leading)), (d) the top quark or top antiquark with the second-largest $p_{T}\left(y_{t}\right.$ (trailing)), and (e) the $t \bar{t}$ system $\left(y_{t \bar{t}}\right)$ at the parton level in the full phase space produced in pp collisions at $\sqrt{s}=13 \mathrm{TeV}$. The solid circles represent the experimental data of the CMS Collaboration in literature [7]; the curves are our results calculated by the three-Gaussian functions. 


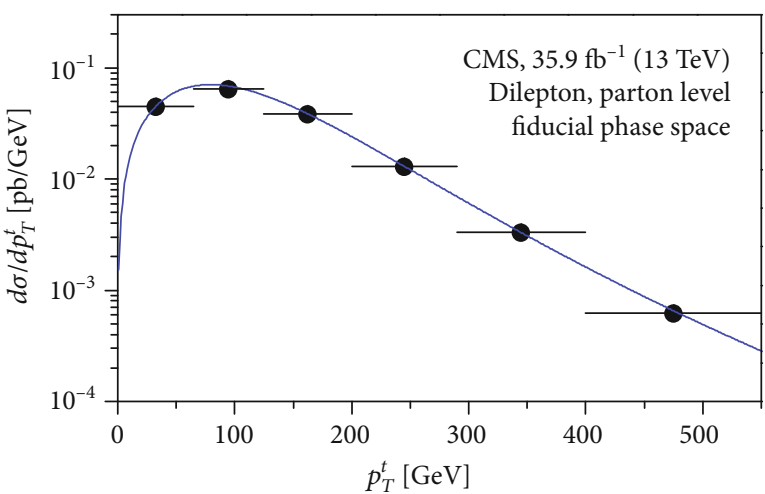

- Data

Tsallis-Pareto-type function

(a)

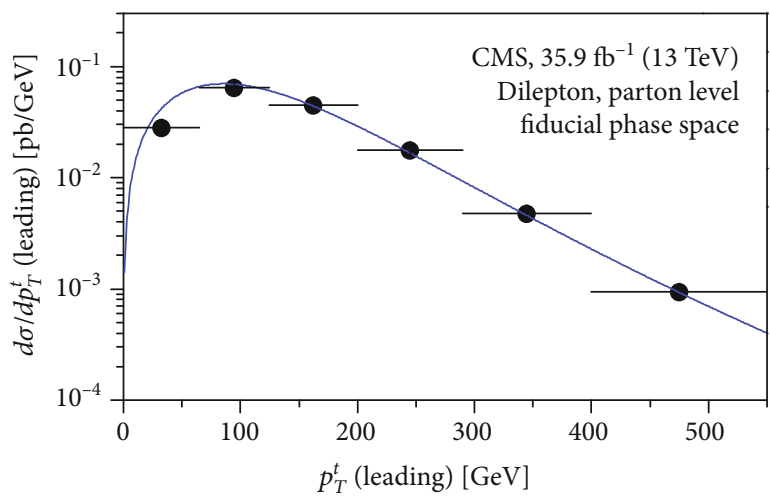

- Data

Tsallis-Pareto-type function

(c)

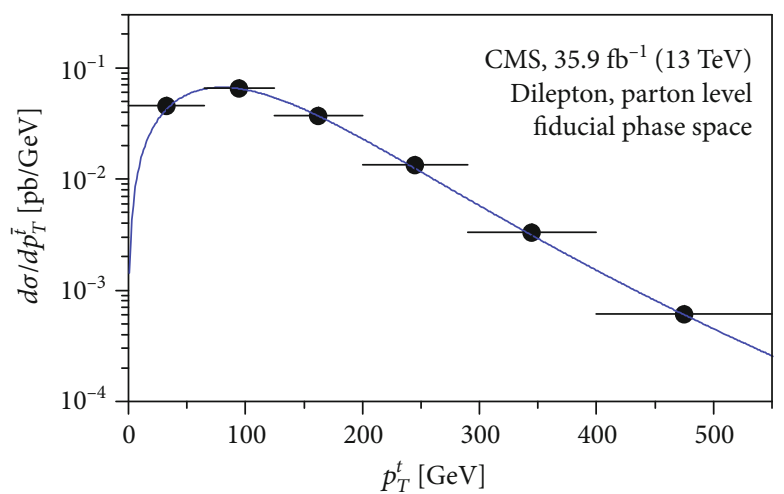

- Data

Tsallis-Pareto-type function

(b)

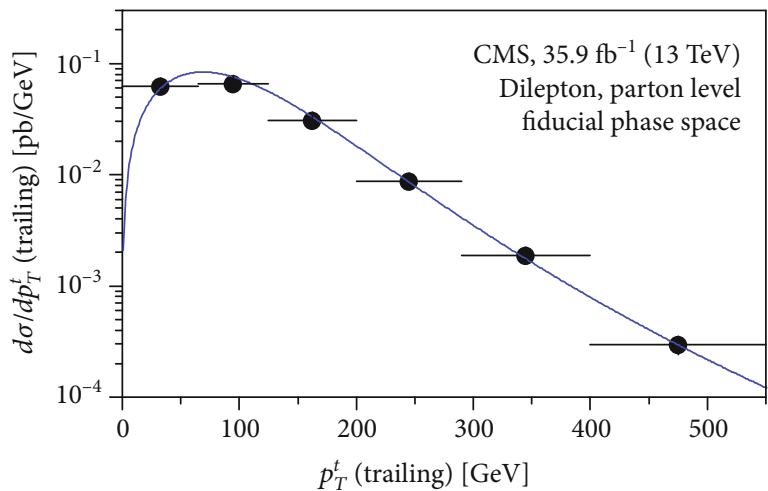

- Data

Tsallis-Pareto-type function

(d)

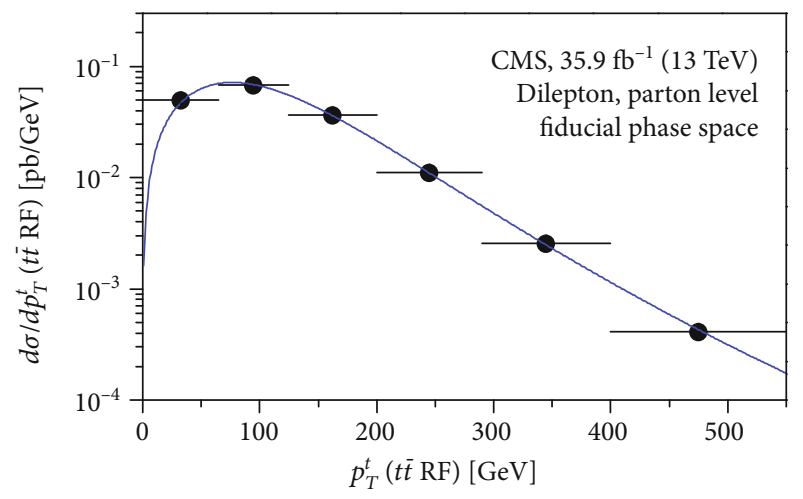

- Data

Tsallis-Pareto-type function

(e)

FIGURE 3: The same as Figure 1 but showing the results of transverse-momentum distribution of (a) $p_{T}^{t}$, (b) $p_{T}^{\bar{t}}$, (c) $p_{T}^{t}$ (leading), (d) $p_{T}^{t}$ (trailing), and (e) $p_{T}^{t}(t \bar{t} \mathrm{RF})$ at the particle level in the fiducial phase space. 


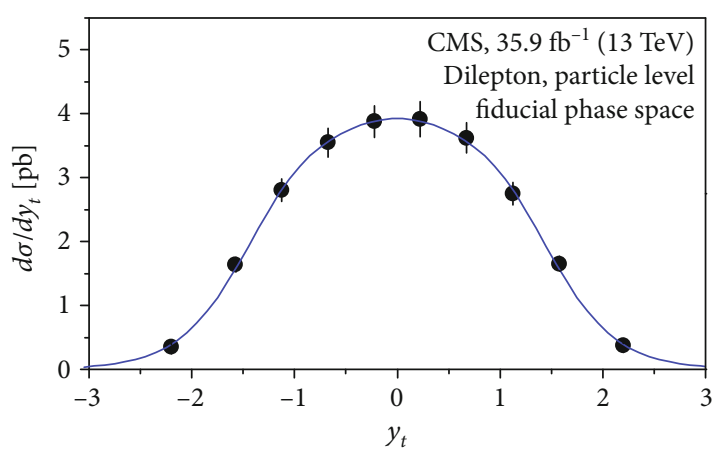

- Data

Three-Gaussian distribution

(a)

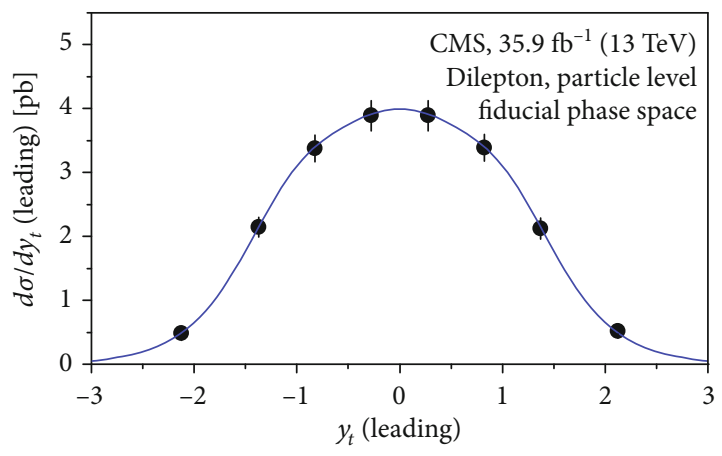

- Data

Three-Gaussian distribution

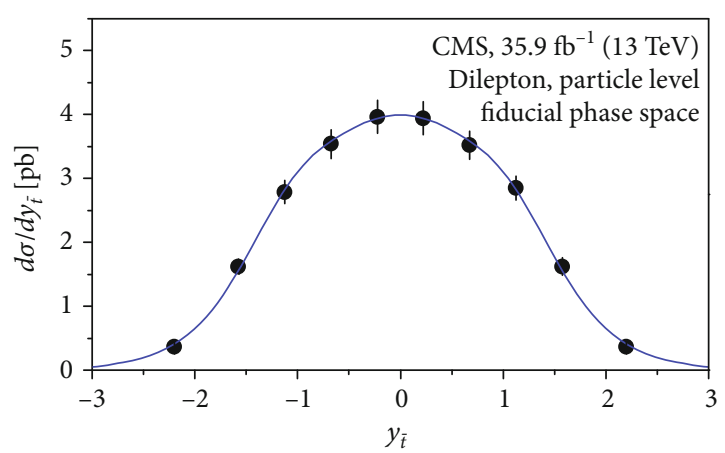

- Data

Three-Gaussian distribution

(b)

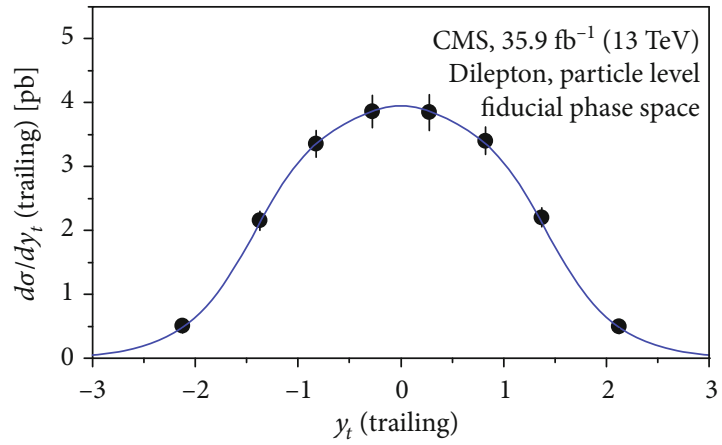

- Data

Three-Gaussian distribution

(d)

(c)

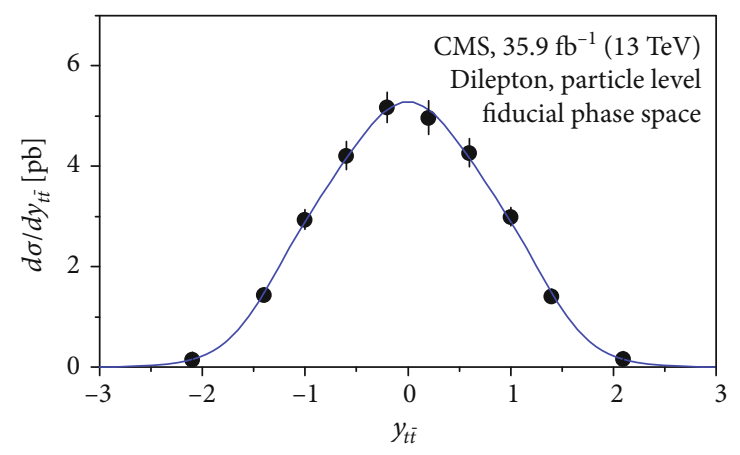

- Data

Three-Gaussian distribution

(e)

FIGURE 4: The same as Figure 2 but showing the results of (a) $y_{t}$, (b) $y_{\bar{t}}$, (c) $y_{t}$ (leading), (d) $y_{t}$ (trailing), and (e) $y_{t \bar{t}}$ at the particle level in the fiducial phase space. 
TABLE 1: Values of parameters and $\chi^{2} /$ dof corresponding to the curves in Figures 1, 3, 5, 7, 11, 12, 13, 15, and 16.

\begin{tabular}{|c|c|c|c|c|}
\hline Figure & Type & $n$ & $T(\mathrm{GeV})$ & $\chi^{2} /$ dof \\
\hline \multicolumn{5}{|l|}{$13 \mathrm{TeV}$} \\
\hline $1(\mathrm{a})$ & $t$ & $9.23 \pm 0.65$ & $29.26 \pm 2.98$ & 0.021 \\
\hline $1(\mathrm{~b})$ & $\bar{t}$ & $9.32 \pm 0.68$ & $29.02 \pm 2.58$ & 0.012 \\
\hline $1(\mathrm{c})$ & $t$ (leading) & $22.00 \pm 0.93$ & $41.56 \pm 3.54$ & 0.411 \\
\hline $1(\mathrm{~d})$ & $t$ (trailing) & $8.05 \pm 0.53$ & $22.25 \pm 2.13$ & 0.037 \\
\hline $1(\mathrm{e})$ & $t(t \bar{t} \mathrm{RF})$ & $9.22 \pm 0.62$ & $26.25 \pm 2.25$ & 0.022 \\
\hline $3(a)$ & $t$ & $8.80 \pm 0.57$ & $31.00 \pm 2.85$ & 0.018 \\
\hline $3(b)$ & $\bar{t}$ & $9.20 \pm 0.65$ & $31.50 \pm 3.00$ & 0.083 \\
\hline $3(c)$ & $t$ (leading) & $10.20 \pm 0.70$ & $36.00 \pm 2.95$ & 0.732 \\
\hline $3(\mathrm{~d})$ & $t$ (trailing) & $8.20 \pm 0.52$ & $24.00 \pm 2.20$ & 0.150 \\
\hline $3(e)$ & $t(t \bar{t} \mathrm{RF})$ & $9.50 \pm 0.67$ & $29.20 \pm 2.50$ & 0.030 \\
\hline $5(a)$ & $l$ & $4.72 \pm 1.00$ & $8.50 \pm 1.30$ & 0.056 \\
\hline $5(\mathrm{~b})$ & $\bar{l}$ & $4.80 \pm 1.10$ & $8.55 \pm 1.30$ & 0.036 \\
\hline $5(c)$ & $l$ (leading) & $5.80 \pm 1.25$ & $13.00 \pm 2.60$ & 3.517 \\
\hline $5(d)$ & $l$ (trailing) & $4.15 \pm 0.85$ & $3.90 \pm 0.70$ & 0.324 \\
\hline $5(e)$ & $\overline{l l}$ & $7.88 \pm 1.55$ & $14.50 \pm 3.00$ & 0.611 \\
\hline $7(\mathrm{a})$ & $b$ (leading) & $15.00 \pm 2.00$ & $28.00 \pm 3.00$ & 6.550 \\
\hline $7(b)$ & $b$ (trailing) & $10.00 \pm 1.30$ & $15.20 \pm 2.30$ & 1.101 \\
\hline 7(c) & $b \bar{b}$ & $23.00 \pm 2.80$ & $30.00 \pm 3.00$ & 13.903 \\
\hline \multicolumn{5}{|l|}{$7 \mathrm{TeV}$} \\
\hline $11(\mathrm{a})$ & $t$ & $9.52 \pm 0.68$ & $25.00 \pm 2.50$ & 0.024 \\
\hline $11(\mathrm{c})$ & $\bar{t}$ & $1.40 \pm 0.15$ & $0.27 \pm 0.07$ & 0.078 \\
\hline $12(\mathrm{a})$ & $b$ & $21.40 \pm 1.80$ & $28.80 \pm 2.80$ & 0.406 \\
\hline $12(\mathrm{c})$ & $l$ & $3.95 \pm 0.40$ & $7.30 \pm 0.90$ & 0.104 \\
\hline \multicolumn{5}{|l|}{$8 \mathrm{TeV}$} \\
\hline 13(a) & $t$ & $8.96 \pm 0.86$ & $26.82 \pm 2.52$ & 0.064 \\
\hline $13(b)$ & $t \bar{t}$ & $2.32 \pm 0.30$ & $3.10 \pm 0.40$ & 1.206 \\
\hline $13(\mathrm{c})$ & $t$ (leading) & $13.54 \pm 1.16$ & $33.68 \pm 2.80$ & 1.144 \\
\hline $13(d)$ & $t$ (trailing) & $7.12 \pm 0.82$ & $20.23 \pm 2.10$ & 0.061 \\
\hline $13(\mathrm{e})$ & $t(t \bar{t} \mathrm{RF})$ & $9.35 \pm 0.90$ & $24.45 \pm 2.50$ & 0.116 \\
\hline $15(\mathrm{a})$ & $l$ & $4.97 \pm 0.57$ & $8.50 \pm 0.50$ & 59.839 \\
\hline $15(b)$ & $\overline{l l}$ & $6.80 \pm 0.80$ & $12.60 \pm 1.10$ & 1.144 \\
\hline $16(\mathrm{a})$ & $b$ & $16.95 \pm 1.25$ & $25.58 \pm 2.53$ & 0.988 \\
\hline $16(b)$ & $b \bar{b}$ & $16.32 \pm 1.08$ & $29.06 \pm 2.86$ & 19.317 \\
\hline
\end{tabular}

[5]. So, in this work, we chose the Tsallis-Pareto-type function to describe the transverse momentum spectra. The Tsallis-Pareto-type function can be written as [5]

$$
\frac{d^{2} N}{d y d p_{T}}=\frac{d N}{d y} C p_{T}\left[1+\frac{m_{T}-m_{0}}{n T}\right]^{-n}
$$

where $m_{0}$ is the rest mass of each particle; $C$ and $m_{T}$ are given by

$$
\begin{gathered}
C=\frac{(n-1)(n-2)}{n T\left[n T+(n-2) m_{0}\right]}, \\
m_{T}=\sqrt{m_{0}^{2}+p_{T}^{2}} .
\end{gathered}
$$

$T$ and $n$ are free parameters. $T$ means the mean effective temperature of the interacting system, and it is connected with the average particle energy. $n$ reveals the nonextensivity of the process, and it denotes the departure of the spectra from the Boltzmann distribution [5]. We could obtain values of the two free parameters ( $T$ and $n$ ) by using the Tsallis-Pareto-type function to fit the $p_{T}$ spectra.

2.2. The Three-Source Landau Hydrodynamic Model. The three-source Landau hydrodynamic model has become a mature theoretical model in research for the pseudorapidity distributions at nuclear collisions. In our previous work, this model has described the experimental data successfully [6]. In the present work, we will use it to fit the pseudorapidity distributions of particles (top quark, lepton, and bottom quark) produced in pp collisions at $\sqrt{s}=13 \mathrm{TeV}$ again. The following is a brief description of the three-source Landau hydrodynamic model.

The source means particle emission source. We think the rapidity distribution of particles produced in highenergy collisions is contributed by the three emission sources. The three emission sources are a central source $(C)$, a target source $(T)$, and a projectile $(P)$. The central source is located at the central of rapidity distribution and covers the whole rapidity range. The target source and projectile source are located at the left and right side of the central source, respectively. And the target source and projectile source are revisions for the central source. For the rapidity distribution of particles produced in each emission source, we can use a Gaussian form of the Landau solution to describe it [23-25]:

$$
\frac{d N_{c h}}{d y}=\frac{N_{0}}{\sqrt{2 \pi} \sigma_{X}} \exp \left(-\frac{\left(y-y_{X}\right)^{2}}{2 \sigma_{X}^{2}}\right),
$$

where $N_{0}$ is the normalization constant, $y$ is rapidity, $\sigma$ denote the width of rapidity distribution, and $X$ represents the type of emission source.

Actually, the three-source Landau hydrodynamic model can be written as a form of a superposition of threeGaussian forms of the Landau solution:

$$
\begin{aligned}
\frac{d N_{c h}}{d y}= & \frac{N_{0}}{\sqrt{2 \pi}}\left\{\frac{k_{T}}{\sigma_{T}} \exp \left(-\frac{\left(y-y_{T}\right)^{2}}{2 \sigma_{T}^{2}}\right)+\frac{k_{C}}{\sigma_{C}} \exp \right. \\
& \left.\cdot\left(-\frac{\left(y-y_{C}\right)^{2}}{2 \sigma_{C}^{2}}\right)+\frac{k_{P}}{\sigma_{P}} \exp \left(-\frac{\left(y-y_{P}\right)^{2}}{2 \sigma_{P}^{2}}\right)\right\} .
\end{aligned}
$$


TABLE 2: Values of parameters and $\chi^{2} /$ dof corresponding to the curves in Figures $2,4,6,8,11,12,14,15$, and 16.

\begin{tabular}{|c|c|c|c|c|c|c|}
\hline Figure & Type & $k_{\mathrm{C}}$ & $y_{P} / \eta_{P}$ & $\sigma(C)$ & $\sigma(P)$ & $\chi^{2} /$ dof \\
\hline \multicolumn{7}{|l|}{$13 \mathrm{TeV}$} \\
\hline $2(a)$ & $t$ & $0.887 \pm 0.017$ & $1.354 \pm 0.074$ & $1.230 \pm 0.025$ & $0.718 \pm 0.015$ & 0.013 \\
\hline 2(b) & $\bar{t}$ & $0.892 \pm 0.020$ & $1.354 \pm 0.074$ & $1.230 \pm 0.025$ & $0.718 \pm 0.018$ & 0.026 \\
\hline $2(c)$ & $t$ (leading) & $0.908 \pm 0.022$ & $1.354 \pm 0.074$ & $1.245 \pm 0.030$ & $0.725 \pm 0.018$ & 0.019 \\
\hline $2(d)$ & $t$ (trailing) & $0.892 \pm 0.018$ & $1.354 \pm 0.074$ & $1.265 \pm 0.033$ & $0.720 \pm 0.015$ & 0.012 \\
\hline $2(\mathrm{e})$ & $t \bar{t}$ & $0.935 \pm 0.025$ & $1.354 \pm 0.074$ & $1.040 \pm 0.025$ & $0.518 \pm 0.008$ & 0.020 \\
\hline $4(\mathrm{a})$ & $t$ & $0.840 \pm 0.020$ & $1.100 \pm 0.060$ & $1.000 \pm 0.020$ & $0.470 \pm 0.006$ & 0.037 \\
\hline $4(b)$ & $\bar{t}$ & $0.875 \pm 0.022$ & $1.100 \pm 0.060$ & $1.020 \pm 0.020$ & $0.425 \pm 0.008$ & 0.102 \\
\hline $4(\mathrm{c})$ & $t$ (leading) & $0.875 \pm 0.022$ & $1.100 \pm 0.060$ & $1.020 \pm 0.022$ & $0.425 \pm 0.007$ & 0.053 \\
\hline $4(d)$ & $t$ (trailing) & $0.875 \pm 0.025$ & $1.100 \pm 0.060$ & $1.020 \pm 0.020$ & $0.425 \pm 0.008$ & 0.032 \\
\hline $4(\mathrm{e})$ & $t \bar{t}$ & $0.905 \pm 0.027$ & $1.100 \pm 0.060$ & $0.780 \pm 0.018$ & $0.355 \pm 0.005$ & 0.232 \\
\hline $6(\mathrm{a})$ & $l$ & $0.915 \pm 0.103$ & $1.225 \pm 0.300$ & $1.235 \pm 0.040$ & $0.650 \pm 0.035$ & 0.027 \\
\hline $6(b)$ & $\bar{l}$ & $0.912 \pm 0.100$ & $1.225 \pm 0.300$ & $1.235 \pm 0.040$ & $0.672 \pm 0.040$ & 0.014 \\
\hline $6(c)$ & $l$ (leading) & $0.950 \pm 0.110$ & $0.990 \pm 0.285$ & $1.235 \pm 0.030$ & $0.560 \pm 0.030$ & 0.033 \\
\hline $6(d)$ & $l$ (trailing) & $0.918 \pm 0.105$ & $1.265 \pm 0.310$ & $1.268 \pm 0.035$ & $0.670 \pm 0.040$ & 0.040 \\
\hline $8(a)$ & $b$ (leading) & $0.930 \pm 0.110$ & $1.225 \pm 0.280$ & $1.210 \pm 0.050$ & $0.650 \pm 0.045$ & 0.012 \\
\hline $8(b)$ & $b$ (trailing) & $0.915 \pm 0.105$ & $1.550 \pm 0.300$ & $1.270 \pm 0.050$ & $0.680 \pm 0.060$ & 0.012 \\
\hline \multicolumn{7}{|l|}{$7 \mathrm{TeV}$} \\
\hline $11(b)$ & $t$ & $0.880 \pm 0.110$ & $1.290 \pm 0.328$ & $0.970 \pm 0.015$ & $0.430 \pm 0.010$ & 0.088 \\
\hline $11(\mathrm{~d})$ & $t \bar{t}$ & $0.950 \pm 0.130$ & $1.290 \pm 0.315$ & $0.840 \pm 0.010$ & $0.450 \pm 0.013$ & 0.056 \\
\hline $12(\mathrm{~b})$ & $b$ & $0.920 \pm 0.125$ & $1.290 \pm 0.325$ & $1.190 \pm 0.030$ & $0.700 \pm 0.020$ & 0.040 \\
\hline $12(\mathrm{c})$ & $l$ & $0.904 \pm 0.110$ & $1.290 \pm 0.320$ & $1.120 \pm 0.030$ & $0.700 \pm 0.022$ & 0.009 \\
\hline \multicolumn{7}{|l|}{$8 \mathrm{TeV}$} \\
\hline $14(\mathrm{a})$ & $t$ & $0.900 \pm 0.080$ & $1.290 \pm 0.290$ & $1.110 \pm 0.020$ & $0.680 \pm 0.015$ & 0.028 \\
\hline $14(\mathrm{~b})$ & $t \bar{t}$ & $0.900 \pm 0.100$ & $1.290 \pm 0.300$ & $0.875 \pm 0.015$ & $0.540 \pm 0.010$ & 0.070 \\
\hline $15(c)$ & $l$ & $0.905 \pm 0.100$ & $1.280 \pm 0.260$ & $1.140 \pm 0.025$ & $0.570 \pm 0.018$ & 0.232 \\
\hline $16(c)$ & $b$ & $0.923 \pm 0.112$ & $1.280 \pm 0.255$ & $1.240 \pm 0.030$ & $0.550 \pm 0.015$ & 0.027 \\
\hline
\end{tabular}

A given parameter $k$ is the contribution of each emission source, $k_{T}+k_{C}+k_{P}=1$. For the symmetric collision, we think $k_{T}=k_{P}$. Because of $y \approx \eta$ at very high energy, we could describe the pseudorapidity distributions of lepton by the following formula:

$$
\begin{aligned}
\frac{d N_{c h}}{d \eta}= & \frac{N_{0}}{\sqrt{2 \pi}}\left\{\frac{k_{T}}{\sigma_{T}} \exp \left(-\frac{\left(\eta-\eta_{T}\right)^{2}}{2 \sigma_{T}^{2}}\right)+\frac{k_{C}}{\sigma_{C}} \exp \right. \\
& \left.\cdot\left(-\frac{\left(\eta-\eta_{C}\right)^{2}}{2 \sigma_{C}^{2}}\right)+\frac{k_{P}}{\sigma_{P}} \exp \left(-\frac{\left(\eta-\eta_{P}\right)^{2}}{2 \sigma_{P}^{2}}\right)\right\} .
\end{aligned}
$$

Significantly, as a revision of the central source, the contributions of the target source and projectile source are small.

\section{Comparisons with Experimental Data}

From the experimental point of view, the particle-level definition is expected to result in decreased uncertainties with respect to a definition at the parton level [26]. So, in this work, we use the same approach to analyze the experimental data.

In this section, we study the transverse momentum and pseudorapidity spectrum of the top quark, lepton, and $b$ jet in proton-proton collisions at LHC. Based on the TsallisPareto-type function, we describe the transverse momentum spectra of the related product. And we use the three-source Landau hydrodynamic model to analyze the pseudorapidity and rapidity distribution.

Figures 1 and 2 show the transverse momentum and rapidity cross sections of (a) the top quark $(t)$, (b) the top antiquark $(\bar{t}),(\mathrm{c})$ the top quark or top antiquark with 


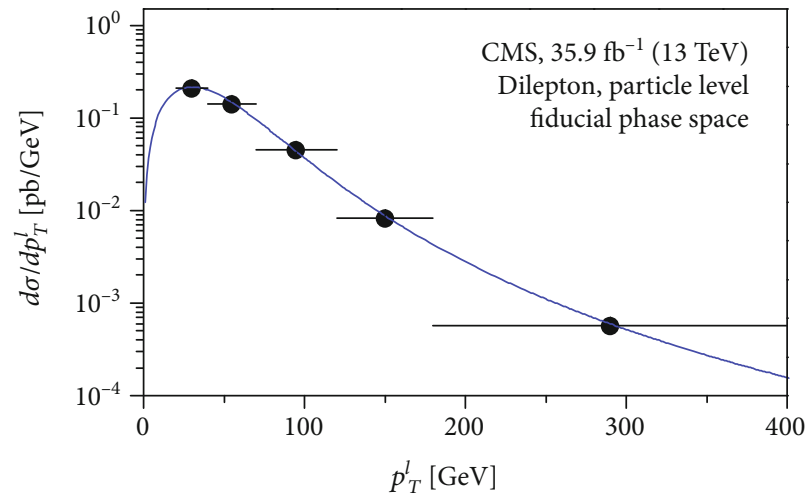

- Data

Tsallis-Pareto-type function

(a)

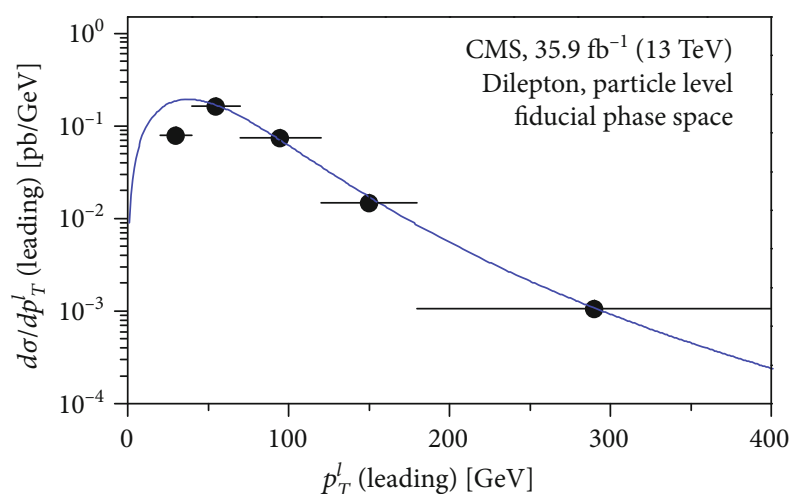

- Data

Tsallis-Pareto-type function

(c)

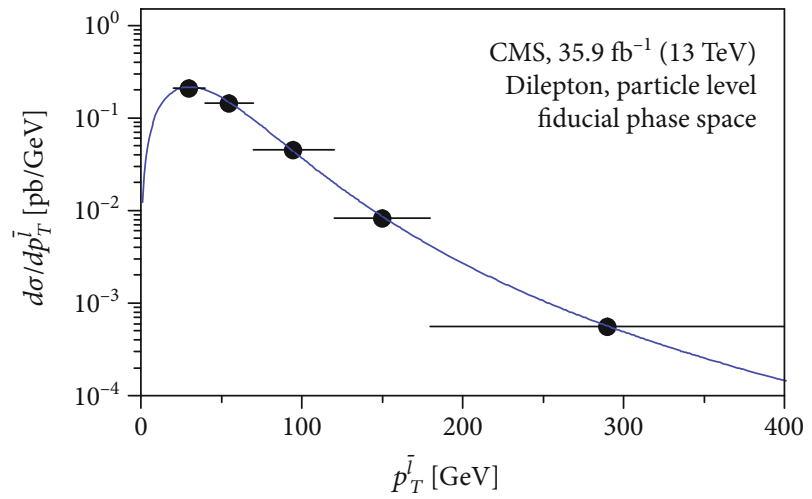

- Data

Tsallis-Pareto-type function

(b)

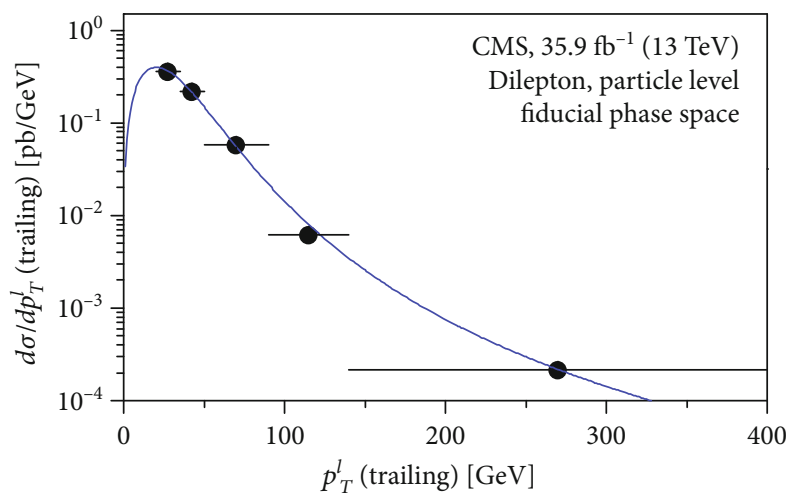

- Data

Tsallis-Pareto-type function

(d)

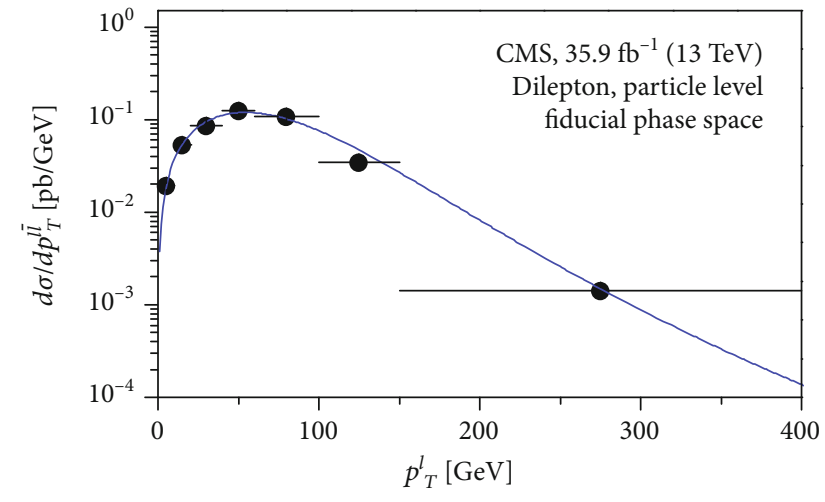

- Data

Tsallis-Pareto-type function

(e)

FIGURE 5: The same as Figure 1 but showing the results of transverse momentum distribution of (a) the lepton $\left(p_{T}^{l}\right)$, (b) the antilepton $\left(p_{T}^{\bar{l}}\right)$, (c) the lepton or antilepton with the largest $p_{T}\left(p_{T}^{l}\right.$ (leading)), (d) the lepton or antilepton with the second-largest $p_{T}\left(p_{T}^{l}(\right.$ trailing)), and (e) the dilepton system $\left(p_{T}^{\bar{l}}\right)$ at the particle level in the fiducial phase space. 


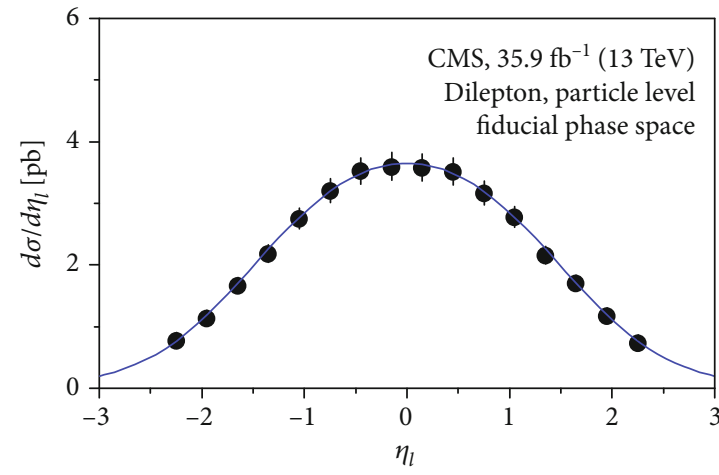

- Data

Three-Gaussian distribution

(a)

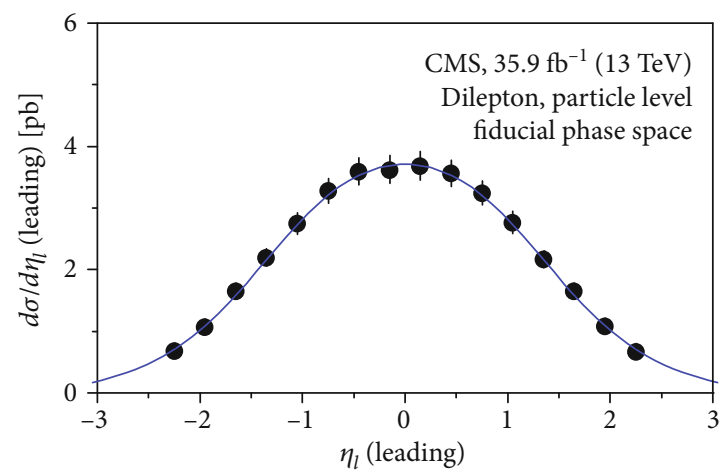

- Data

Three-Gaussian distribution

(c)

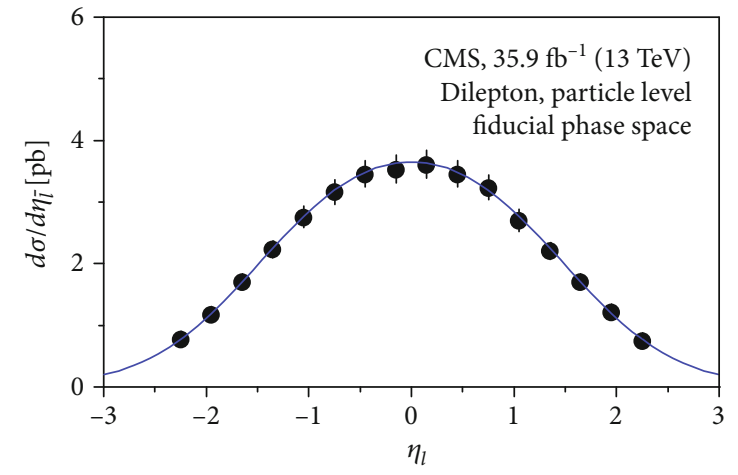

- Data

Three-Gaussian distribution

(b)

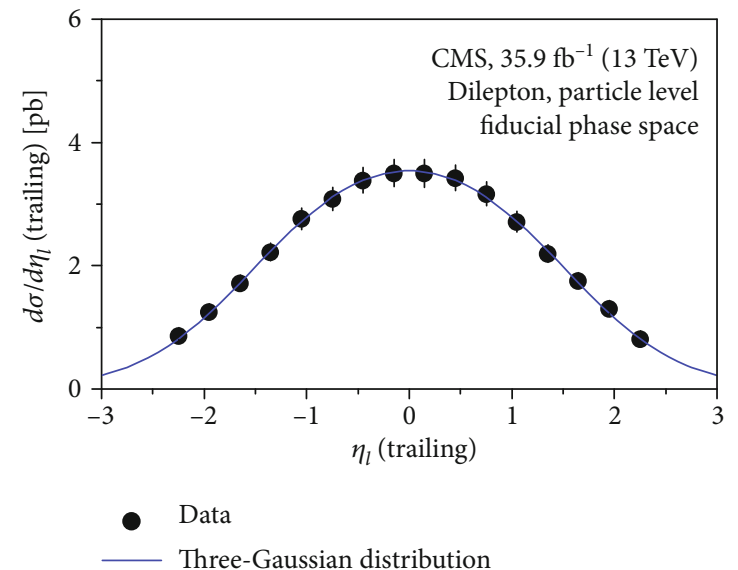

(d)

Figure 6: The same as Figure 2 but showing the results of pseudorapidity distribution of (a) the lepton $\left(\eta_{l}\right)$, (b) the antilepton $\left(\eta_{\bar{l}}\right)$, $(\mathrm{c})$ the lepton or antilepton with the largest $p_{T}\left(\eta_{l}\right.$ (leading)), and (d) the lepton or antilepton with the second-largest $p_{T}\left(\eta_{l}(\operatorname{trailing})\right)$.

the largest $p_{T}$ ( $t$ (leading)), (d) the top quark or top antiquark with the second-largest $p_{T}$ ( $t$ (trailing)), and (e) the top quark in the rest frame of the $t \bar{t}$ system $(t \bar{t} \mathrm{RF})$ at the parton level in the full phase space produced in pp collisions at $\sqrt{s}=13 \mathrm{TeV}$. The same as Figures 1 and 2, but Figures 3 and 4 show the results at the particle level in the fiducial phase space. In these four figures, the solid circles represent the experimental data recorded by the CMS experiment at the LHC and correspond to an integrated luminosity of $35.9 \mathrm{fb}^{-1}$ in 2016 [7]. In Figures 1 and 3 , the curves are our results calculated by the Tsallis-Pareto-type function. In Figures 2 and 4, the curves show the calculate results of the three-Gaussian functions. Obviously, our results fit well with the experimental data. One can see that the Tsallis-Pareto-type function and the three-Gaussian functions are very helpful approaches to describe the transverse momentum and rapidity cross sections of particles, respectively. The related parameter values $\left(n, T, k, y_{P}, \sigma\right)$ extracted from the transverse momentum and rapidity cross sections and $\chi^{2} /$ dof are given in Tables 1 and 2 . We could find that the values of $n$ and $T$ of $t$ (leading) are the biggest in the five-type particles at the parton and particle level in the full and fiducial phase space. The values of $y_{P}$ are the same in the scope of consideration. The values of $k$, $\sigma(C)$, and $\sigma(P)$ are basically the same within the margin of error in the $t, \bar{t}, t$ (leading), and $t$ (trailing), but these three parameter values decreased significantly in the rest frame of the $t \bar{t}$ system.

Figures 5 and 6 show the transverse momentum and rapidity cross sections of (a) the lepton $(l)$, (b) the antilepton $(\bar{l})$, (c) the lepton or antilepton with the largest $p_{T}$ ( $l$ (leading)), and (d) the lepton or antilepton with the secondlargest $p_{T}(l$ (trailing)), and Figure 5(e) shows the transverse momentum cross sections of the dilepton system $(\bar{l})$ at the particle level in the fiducial phase space produced in pp collisions at $\sqrt{s}=13 \mathrm{TeV}$. The solid circles and curves represent the experimental data and our calculated results, respectively. One can see that the results calculated by using the theoretical functions are in agreement with the experimental data. The related parameters are extracted and listed in Tables 1 (5(a)-(e)) and 2 (6(a)-(d)). The values of $n$ and $T$ of the $\bar{l}$ 


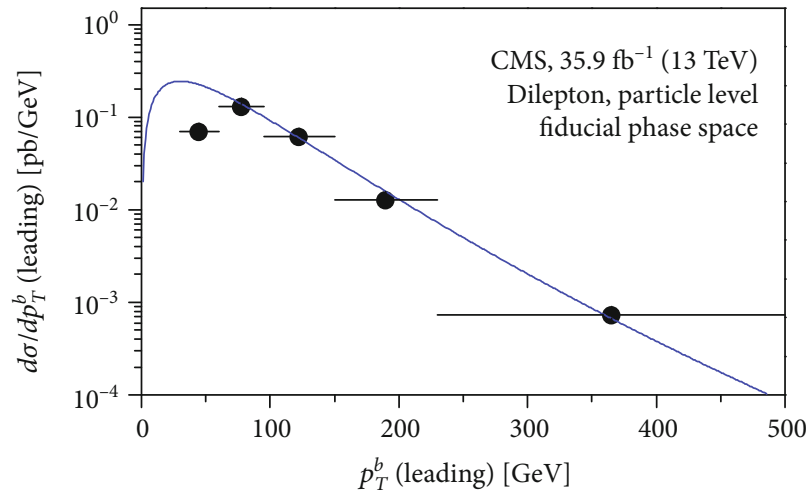

- Data

Tsallis-Pareto-type function

(a)

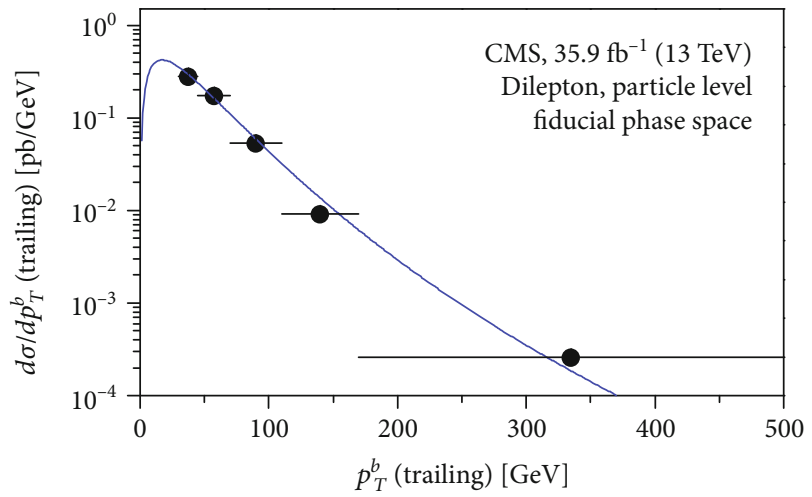

- Data

Tsallis-Pareto-type function

(b)

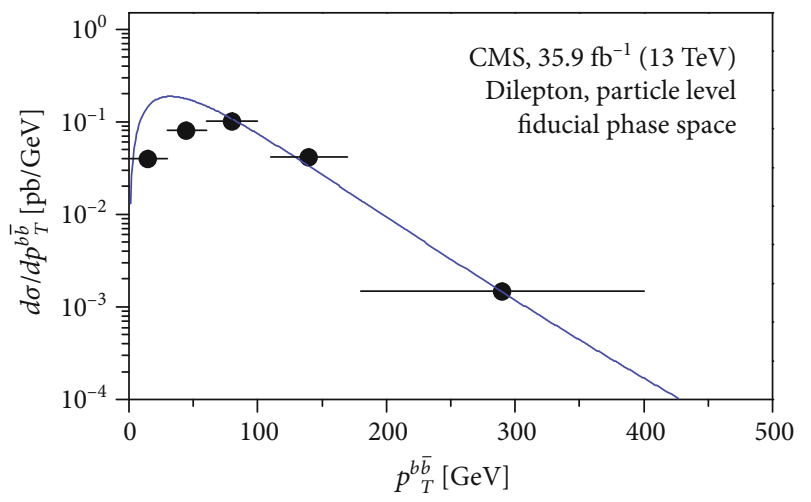

- Data

Tsallis-Pareto-type function

(c)

Figure 7: The same as Figure 1 but showing the results of transverse momentum distribution of (a) the $b$ jet with the largest $p_{T}\left(p_{T}^{b}\right.$ (leading)), (b) the $b$ jet with the second-largest $p_{T}\left(p_{T}^{b}\right.$ (trailing)), and (c) the $b \bar{b}$ system $\left(p_{T}^{b \bar{b}}\right)$ at the particle level in the fiducial phase space.

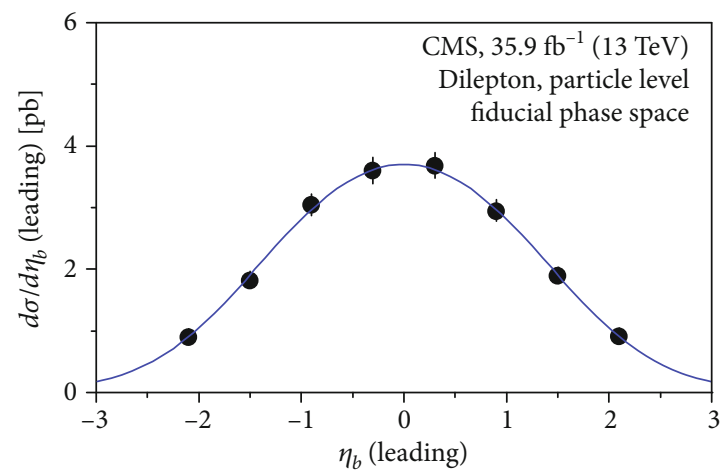

- Data

Three-Gaussian distribution

(a)

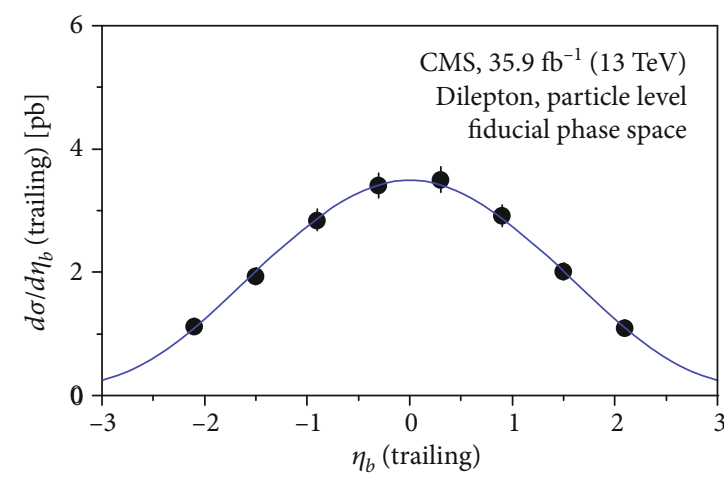

- Data

Three-Gaussian distribution

(b)

FIGURE 8: The same as Figure 2 but showing the results of pseudorapidity distribution of (a) the $b$ jet with the largest $p_{T}\left(\eta_{b}\right.$ (leading)) and (b) the $b$ jet with the second-largest $p_{T}\left(\eta_{b}\right.$ (trailing)). 
TABLE 3: The values of $\left\langle p_{T}\right\rangle$ and $\sqrt{\left\langle p_{T}^{2}\right\rangle / 2}$ are calculated by the curves in Figures $1,3,5$, and 7 .

\begin{tabular}{|c|c|c|c|}
\hline Figure & Type & $\left\langle p_{T}\right\rangle(\mathrm{GeV} / \mathrm{c})$ & $\sqrt{\left\langle p_{T}^{2}\right\rangle / 2}(\mathrm{GeV} / \mathrm{c})$ \\
\hline \multicolumn{4}{|c|}{ At parton level in the full phase space } \\
\hline \multirow{5}{*}{$9(\mathrm{a}) / 10(\mathrm{a})$} & $t$ & $120.691 \pm 0.014$ & $102.471 \pm 6.971$ \\
\hline & $\bar{t}$ & $119.785 \pm 0.012$ & $101.604 \pm 6.247$ \\
\hline & $t$ (leading) & $137.210 \pm 0.015$ & $114.073 \pm 7.430$ \\
\hline & $t$ (trailing) & $103.660 \pm 0.006$ & $88.028 \pm 3.131$ \\
\hline & $t \bar{t}$ & $112.256 \pm 0.008$ & $94.944 \pm 4.114$ \\
\hline \multicolumn{4}{|c|}{ At particle level in the fiducial phase space } \\
\hline \multirow{5}{*}{$9(b) / 10(b)$} & $t$ & $126.834 \pm 0.017$ & $108.302 \pm 8.416$ \\
\hline & $\bar{t}$ & $127.002 \pm 0.018$ & $108.153 \pm 8.762$ \\
\hline & $t$ (leading) & $136.458 \pm 0.022$ & $115.996 \pm 10.800$ \\
\hline & $t$ (trailing) & $108.473 \pm 0.008$ & $92.235 \pm 3.834$ \\
\hline & $t \bar{t}$ & $119.830 \pm 0.012$ & $101.536 \pm 5.958$ \\
\hline \multirow{5}{*}{$9(\mathrm{c}) / 10(\mathrm{c})$} & $l$ & $53.617 \pm 0.004$ & $49.657 \pm 1.768$ \\
\hline & $\bar{l}$ & $53.322 \pm 0.003$ & $49.075 \pm 1.703$ \\
\hline & $l$ (leading) & $64.970 \pm 0.005$ & $58.171 \pm 2.743$ \\
\hline & $l$ (trailing) & $34.914 \pm 0.001$ & $32.255 \pm 0.450$ \\
\hline & $\overline{l l}$ & $81.272 \pm 0.003$ & $68.092 \pm 1.428$ \\
\hline \multirow{3}{*}{$9(\mathrm{~d}) / 10(\mathrm{~d})$} & $b$ (leading) & $69.613 \pm 0.004$ & $62.890 \pm 1.861$ \\
\hline & $b$ (trailing) & $42.804 \pm 0.001$ & $39.883 \pm 0.348$ \\
\hline & $b \bar{b}$ & $69.127 \pm 0.002$ & $61.179 \pm 0.867$ \\
\hline
\end{tabular}

system are bigger than the other particles. The values of $k, y_{P}$, $\sigma(C)$, and $\sigma(P)$ of the considered particles are not different obviously.

The results of transverse momentum and rapidity cross sections of (a) the $b$ jet with the largest $p_{T}$ ( $b$ (leading)) and (b) the $b$ jet with the second-largest $p_{T}$ ( $b$ (trailing)) are showed in Figures 7 and 8, and Figure 7(c) shows the transverse momentum cross sections of the $b \bar{b}$ system at the particle level in the fiducial phase space produced in pp collisions at $\sqrt{s}=13 \mathrm{TeV}$. We have extracted the related parameters by describing the transverse momentum and rapidity cross section distributions and listed them in Tables $1(7(\mathrm{a})-(\mathrm{c}))$ and 2 (8(a)-(b)). The values of parameters are not in an obvious relationship.

Generally, the initial temperature $\left(T_{\mathrm{i}}\right)$ and mean transverse momentum $\left(\left\langle p_{T}\right\rangle\right)$ are important physical quantities to understand the excitation degree of the interacting system. The two physical quantities are not dependent on the selected models and functions; they are only dependent on the experimental data. According to the literatures [27-29], $T_{\mathrm{i}}$ can be described by the ratio of root-mean-square $p_{T}$ to $\sqrt{2}$ $\left(\sqrt{\left\langle p_{T}^{2}\right\rangle / 2}\right)$ approximately. So, we calculated the values of \langle $\left.p_{T}\right\rangle$ and $\sqrt{\left\langle p_{T}^{2}\right\rangle / 2}$ by the curves in Figures $1,3,5$, and 7 and listed them in Table 3. Figures 9 and 10 show the $\left\langle p_{T}\right\rangle$ and $\sqrt{\left\langle p_{T}^{2}\right\rangle / 2}$ of each particle. From Figures 9 and 10, we could observe that the $\left\langle p_{T}\right\rangle$ and $\sqrt{\left\langle p_{T}^{2}\right\rangle / 2}$ of each particle are big. This phenomenon means that the excitation degree of the interacting system is violent.

In order to find the relationship between the free parameter and collision energy, we have extracted the related parameters from the transverse momentum and rapidity cross sections at $7 \mathrm{TeV}$ and $8 \mathrm{TeV}$ (Figures 1116), together. In Figures 11 and 12, the solid circles represent the experimental data recorded by the CMS experiment at $7 \mathrm{TeV}$ and correspond to an integrated luminosity of $5.0 \mathrm{fb}^{-1}$ in 2011 [30]. In Figures 13-16, the solid circles represent the experimental data recorded by the CMS experiment at $8 \mathrm{TeV}$ and correspond to an integrated luminosity of $19.7 \mathrm{fb}^{-1}$ in 2012 [31]. From the transverse momentum and rapidity cross sections of final state particles, we extracted the values of free parameters and listed them in Tables 1 and 2. And we plot the $T, n$, and $\sigma$ values listed in Tables 1 and 2 in Figure 17. In the panel, the symbols represent the values of $T, n$, and $\sigma$, and the lines are linear fitting functions. The intercepts, slopes, and $\chi^{2} /$ dof corresponding to the lines are listed in Table 4. From Figure 17 and Table 4, we found that the values of $T$ show a slight increase with the collision energy increased; this phenomenon may be affected by QGP. It can be explained by the influence of jet quenching effect (quickly energy loss) in the case of high-energy quark and gluon jets penetrating through the dense deconfined matter [32]. $\sigma(C)$ shows to be increased with the collision 


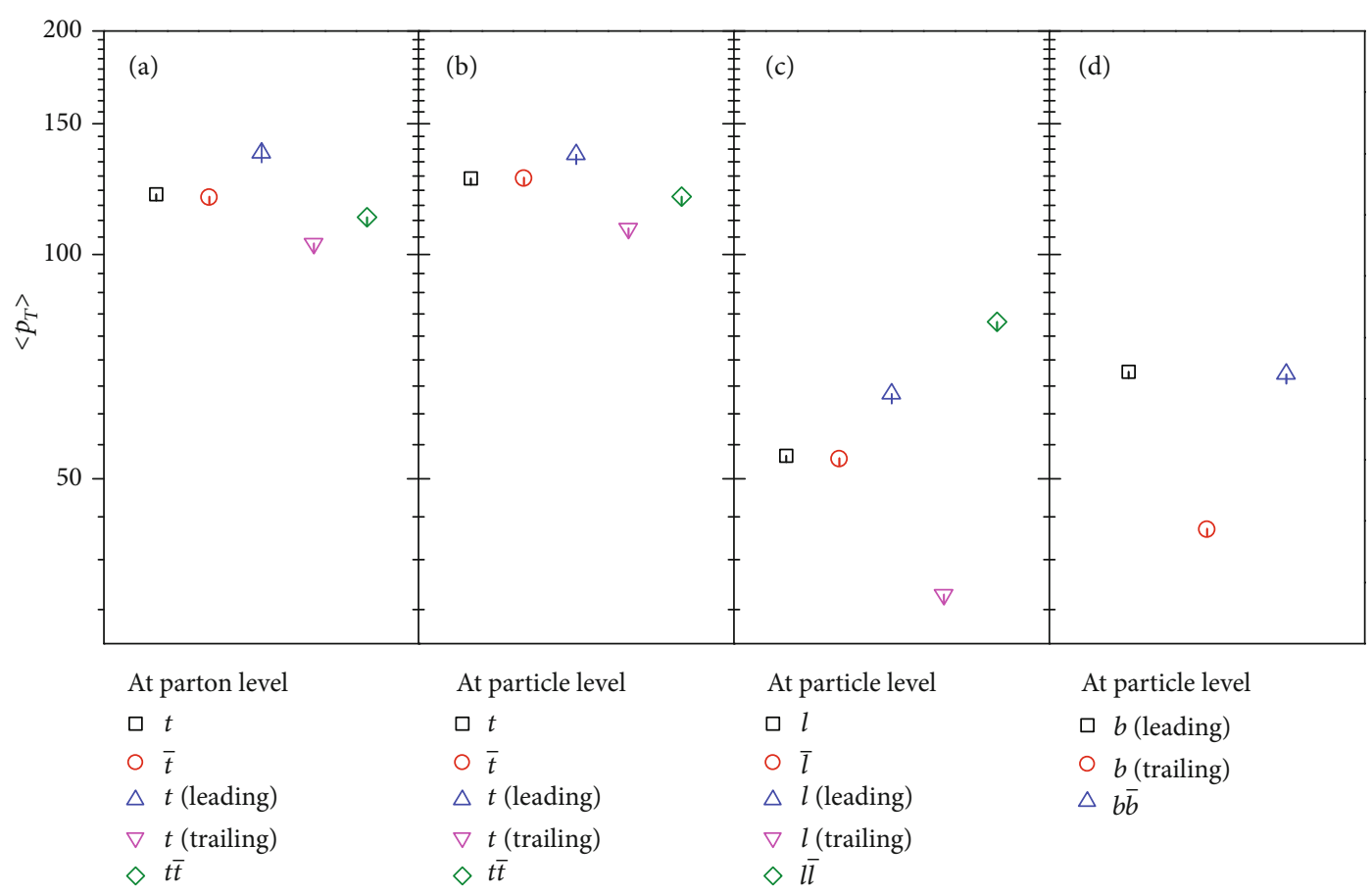

Figure 9: The values of $\left\langle p_{T}\right\rangle$ for different particles.

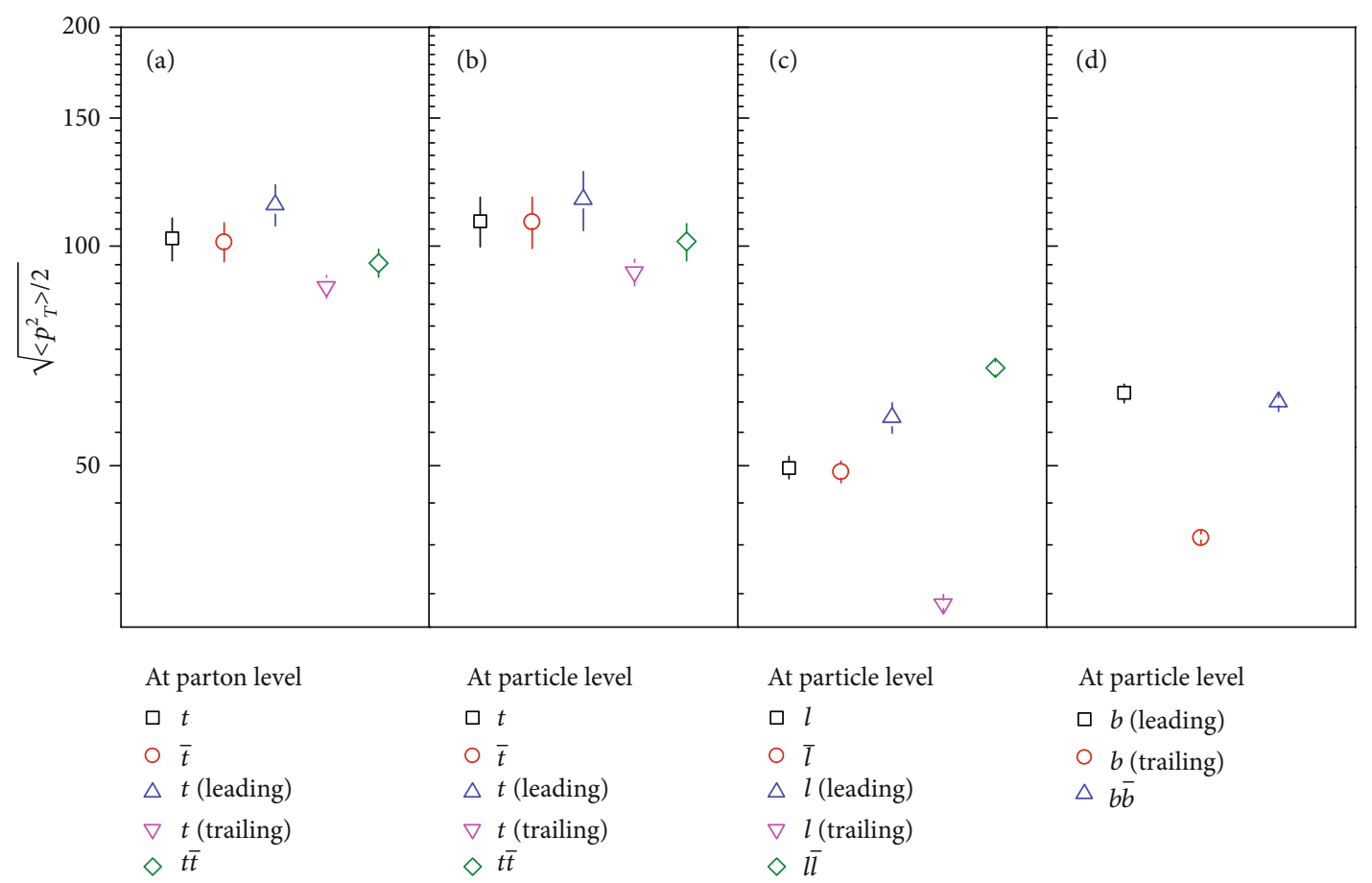

Figure 10: The same as Figure 9 but showing the values of $\sqrt{\left\langle p_{T}^{2}\right\rangle / 2}$. 


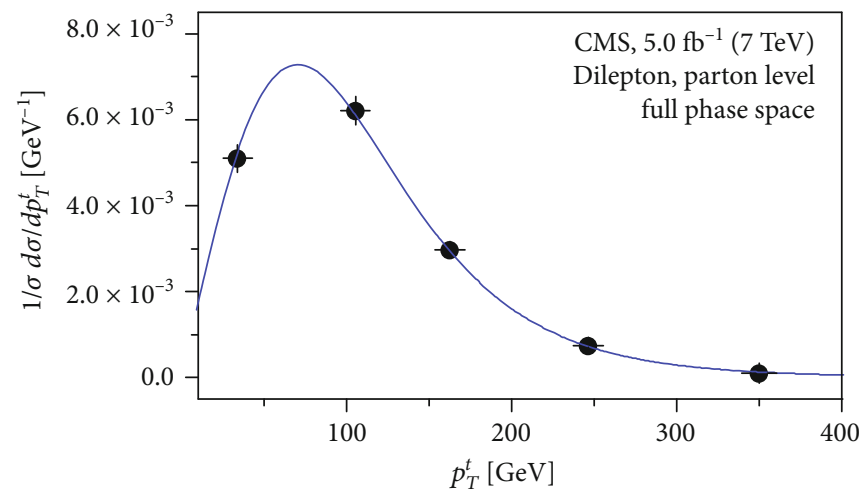

- Data

Tsallis-Pareto-type function

(a)

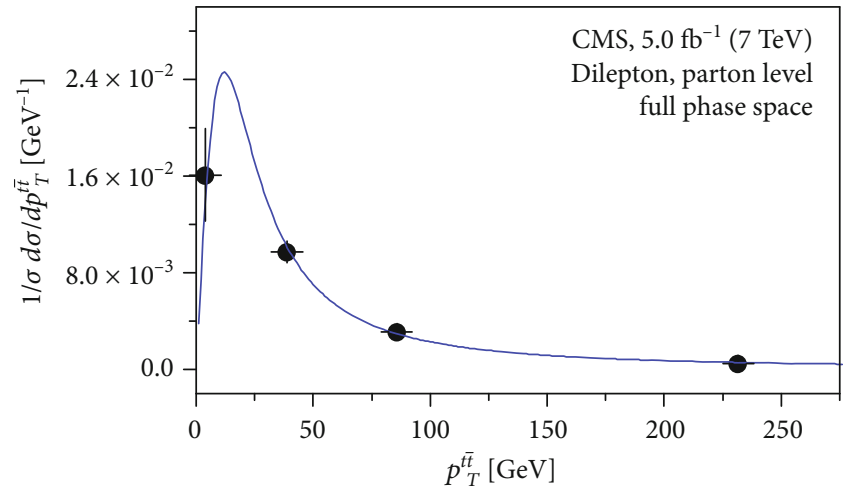

- Data

Tsallis-Pareto-type function

(c)

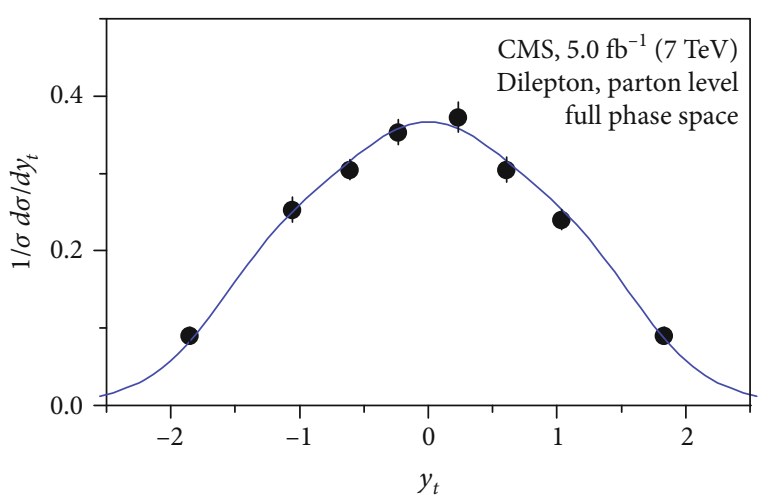

- Data

Three-Gaussian function

(b)

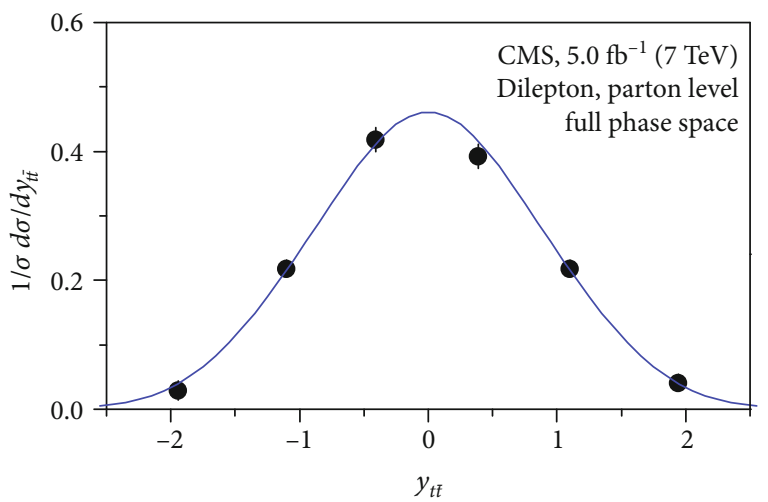

- Data

Three-Gaussian function

(d)

Figure 11: The same as Figures 1 and 2, but showing the results of $t$ and $t \bar{t}$ produced in pp collisions at the parton level in the full phase space at $\sqrt{s}=7 \mathrm{TeV}$. 


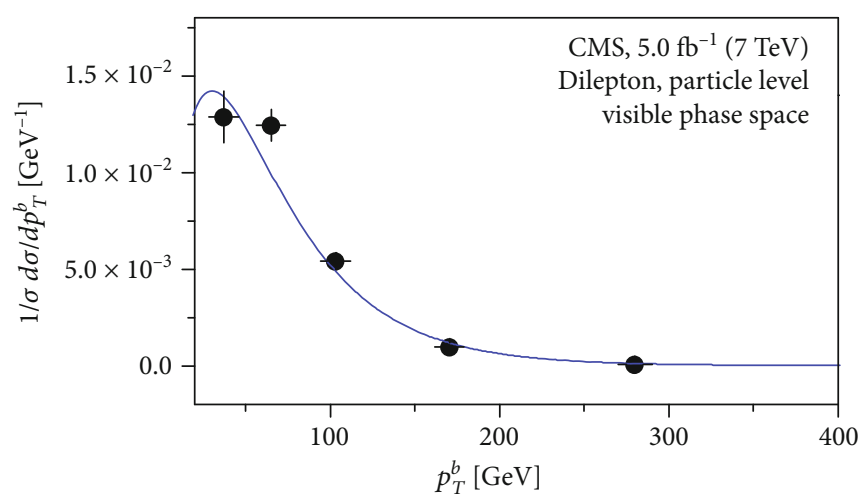

- Data

Tsallis-Pareto-type function

(a)

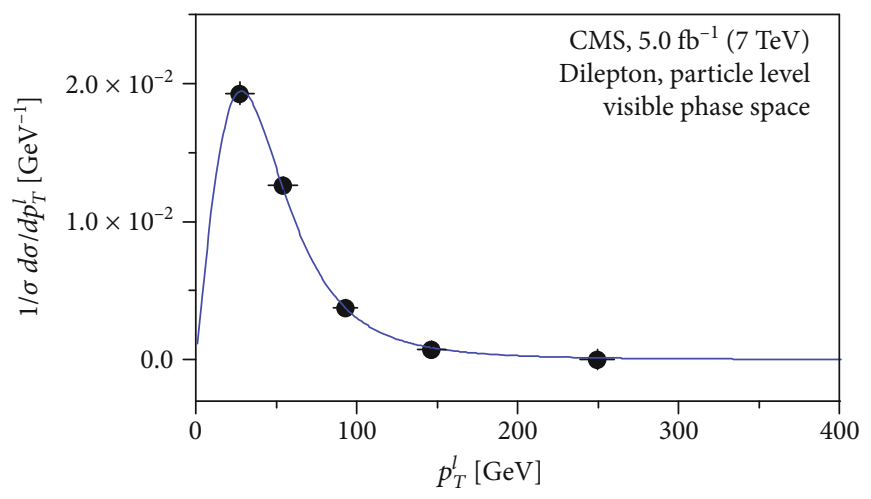

- Data

Tsallis-Pareto-type function

(c)

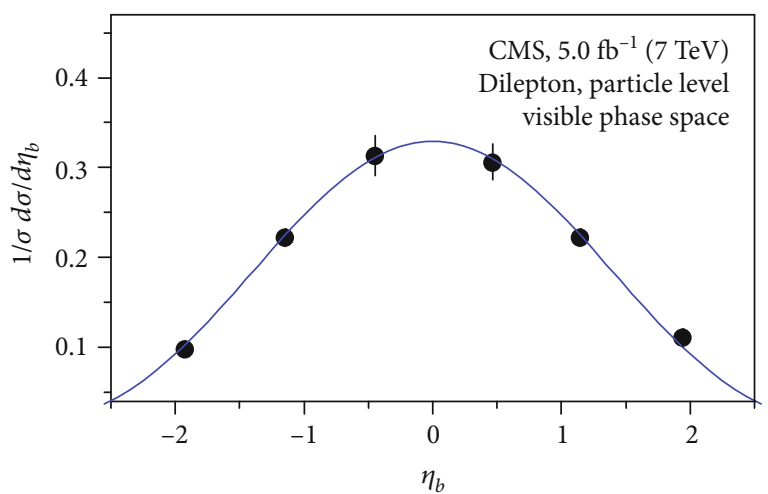

- Data

Three-Gaussian function

(b)

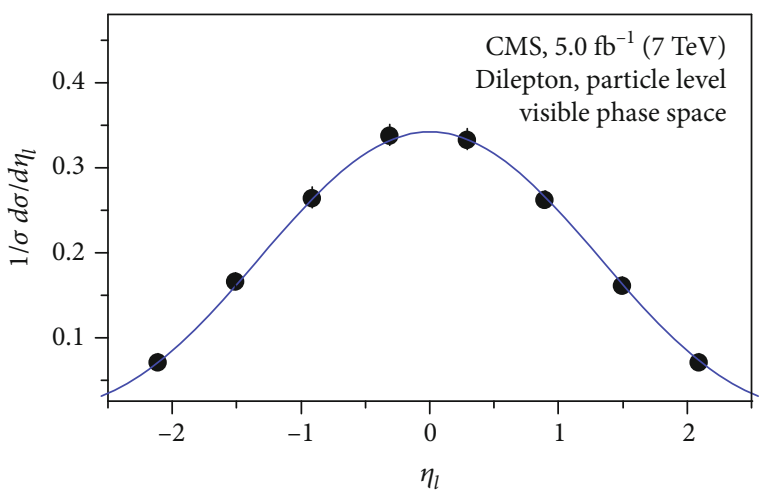

- Data

Three-Gaussian function

(d)

Figure 12: The same as Figures 1 and 5, but showing the results of $b$ and $l$ produced in pp collisions at the particle level in the visible phase space at $\sqrt{s}=7 \mathrm{TeV}$. 


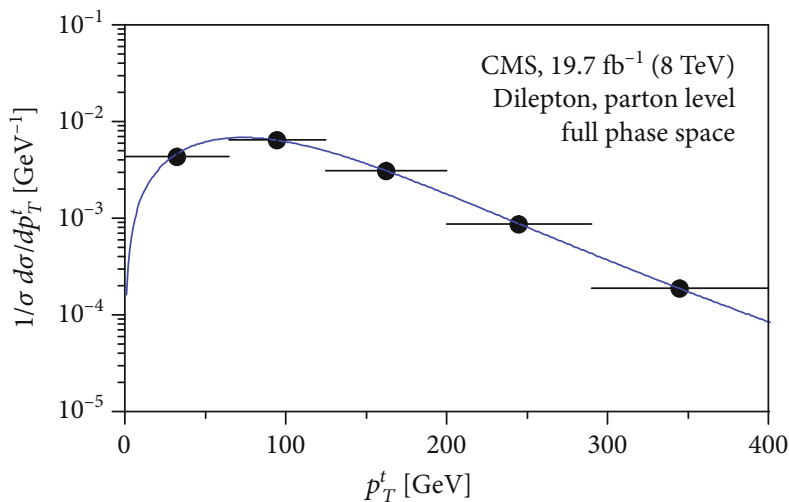

- Data

Tsallis-Pareto-type function

(a)

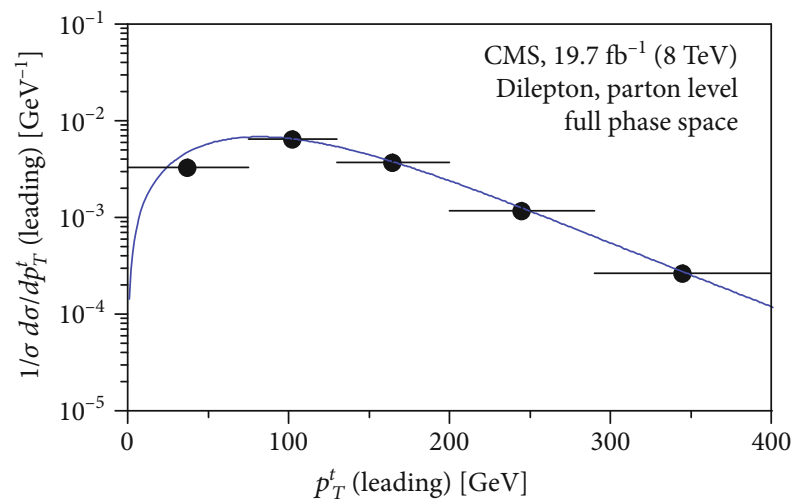

- Data

Tsallis-Pareto-type function

(c)

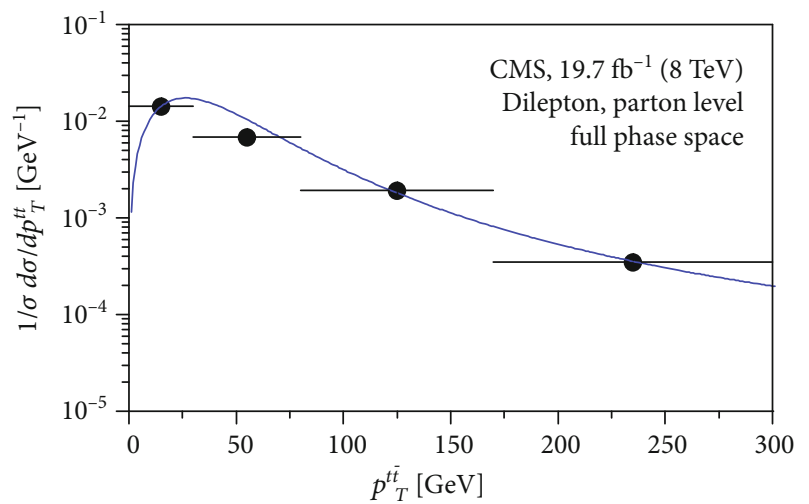

- Data

Tsallis-Pareto-type function

(b)

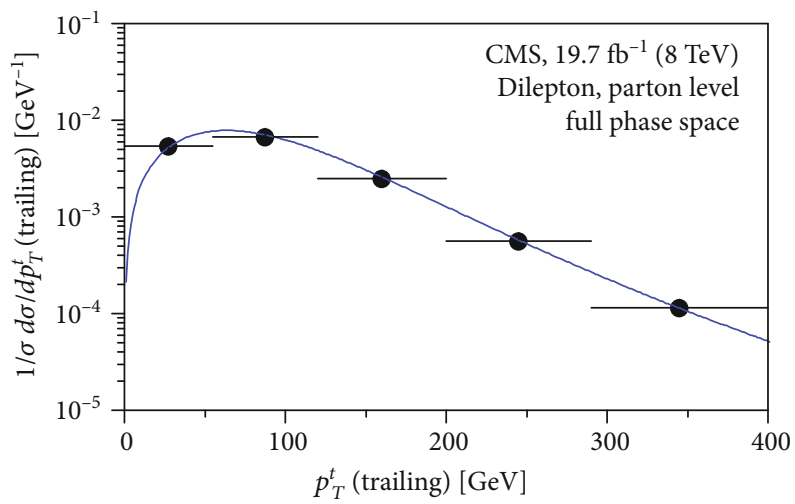

- Data

Tsallis-Pareto-type function

(d)

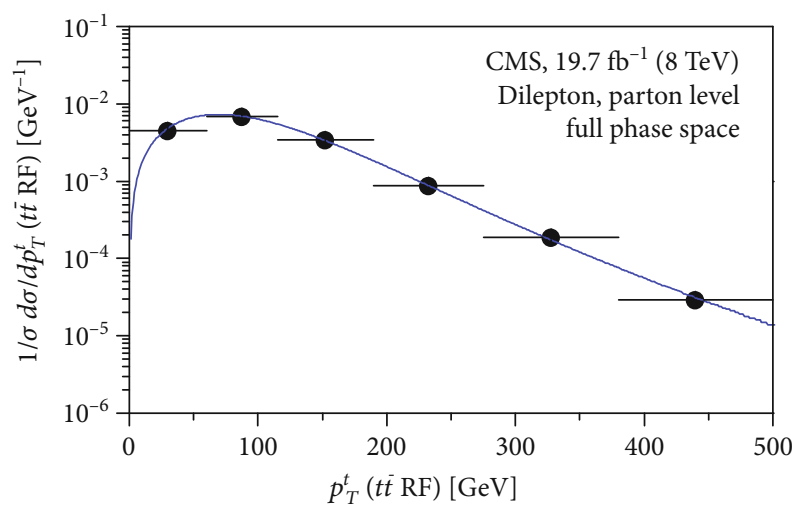

- Data

Tsallis-Pareto-type function

(e)

Figure 13: The same as Figure 1 but showing the results of transverse momentum distribution of (a) $p_{T}^{t}$, (b) $p_{T}^{t \bar{t}}$, (c) $p_{T}^{t}$ (leading), (d) $p_{T}^{t}$ (trailing), and (e) $p_{T}^{t}(t \bar{t} \mathrm{RF})$ at the parton level in the full phase space at $\sqrt{s}=8 \mathrm{TeV}$. 


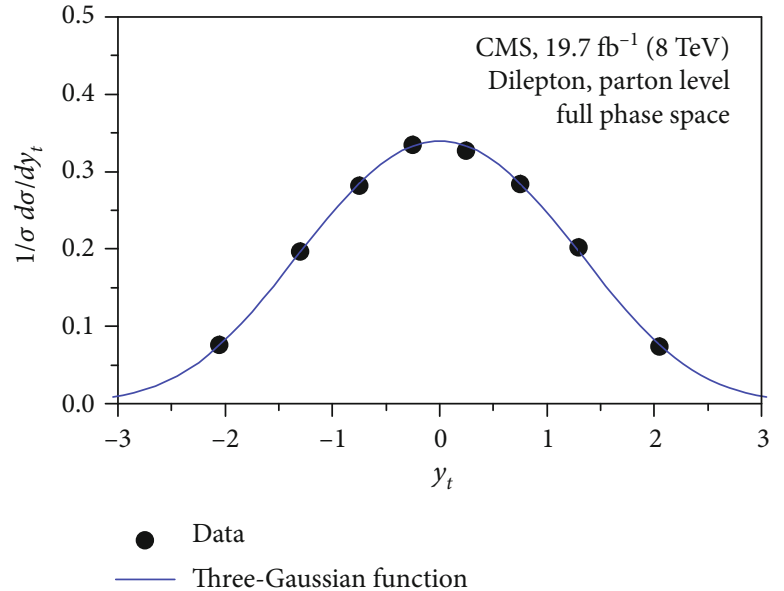

(a)

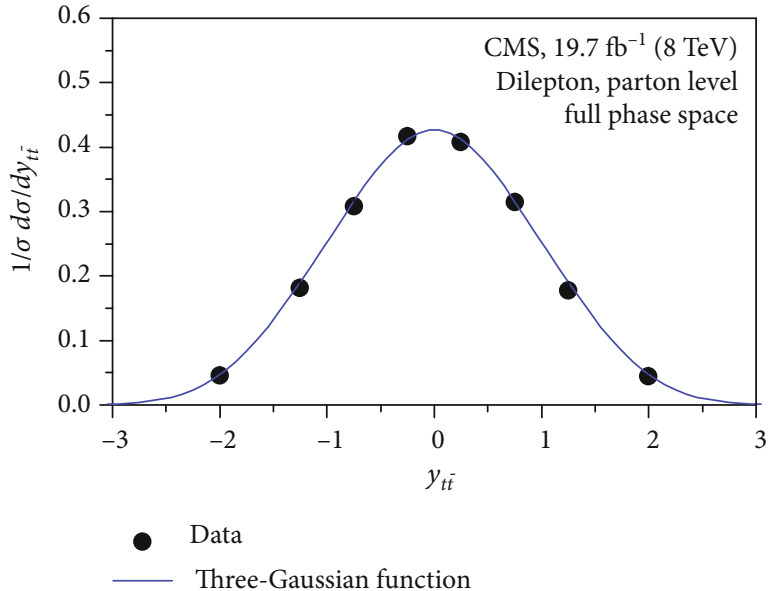

(b)

FIgURE 14: The same as Figure 2 but showing the results of rapidity distribution of $t$ and $t \bar{t}$ at the parton level in the full phase space at $\sqrt{s}=8$ $\mathrm{TeV}$.

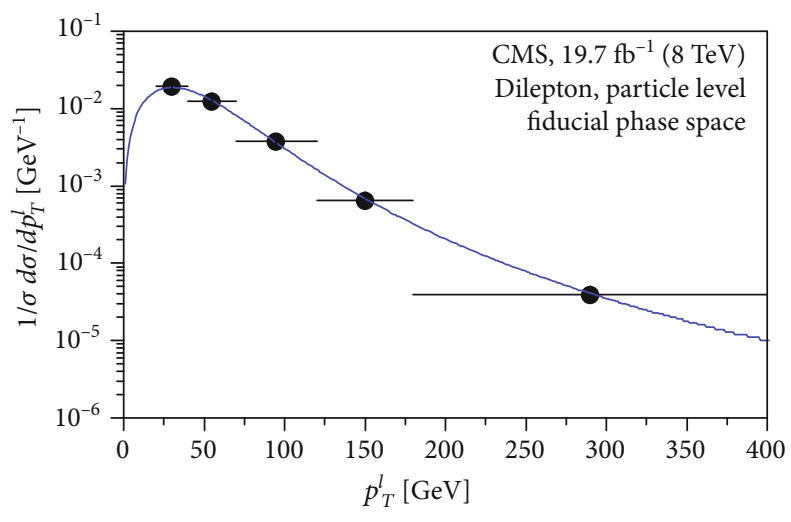

- Data

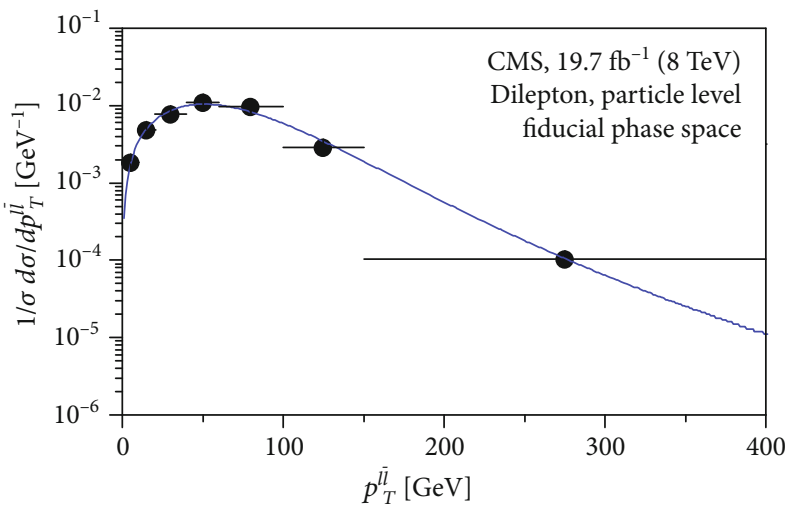

- Data

- Tsallis-Pareto-type function

(b)

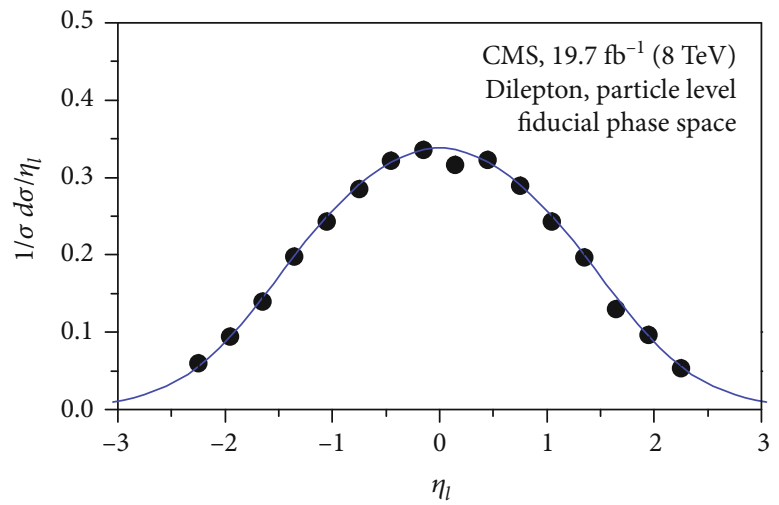

- Data

three-Gaussian function

(c)

Figure 15: The same as Figures 1 and 5, but showing the results of $l$ and $\bar{l}$ produced in pp collisions at the particle level in the fiducial phase space at $\sqrt{s}=8 \mathrm{TeV}$. 


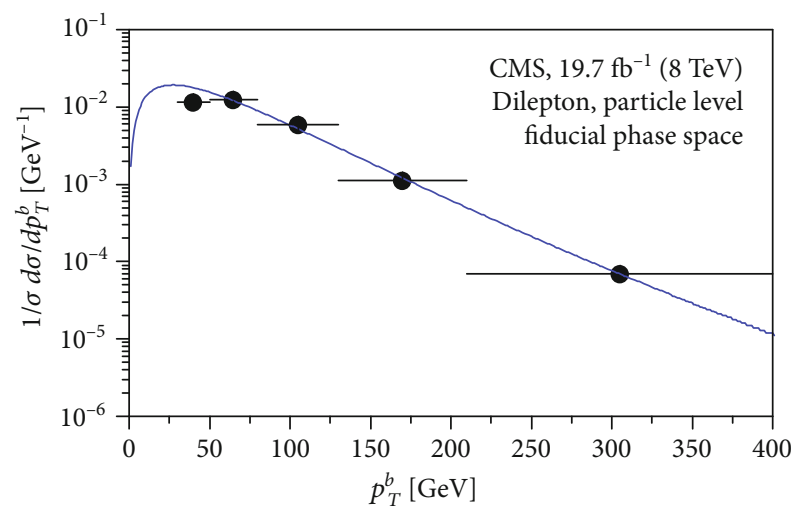

- Data

Tsallis-Pareto-type function

(a)

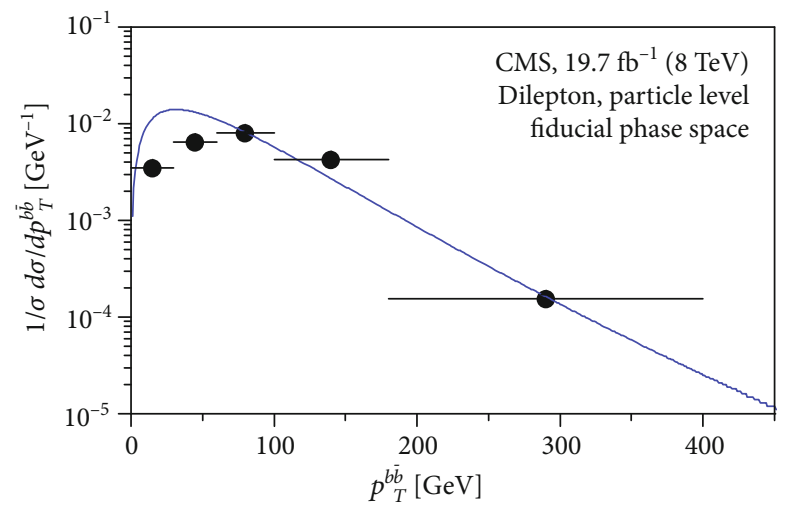

- Data

Tsallis-Pareto-type function

(b)

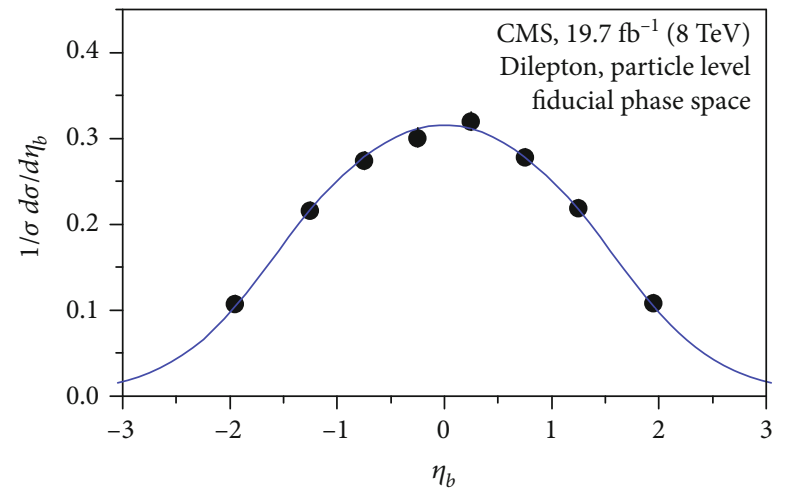

- Data

Three-Gaussian function

(c)

FIGURE 16: The same as Figures 1 and 5, but showing the results of $b$ and $b \bar{b}$ produced in pp collisions at the particle level in the fiducial phase space at $\sqrt{s}=8 \mathrm{TeV}$. 

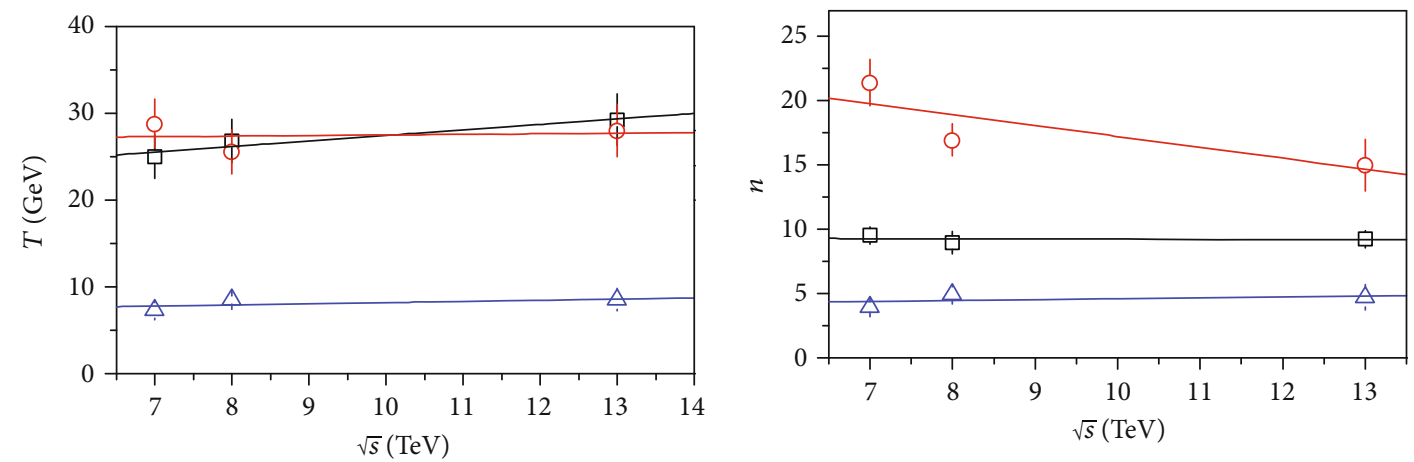
$\square t$
$\circ b$
$\triangle l$

$\square t$

$\circ b$

$\triangle l$

(a)
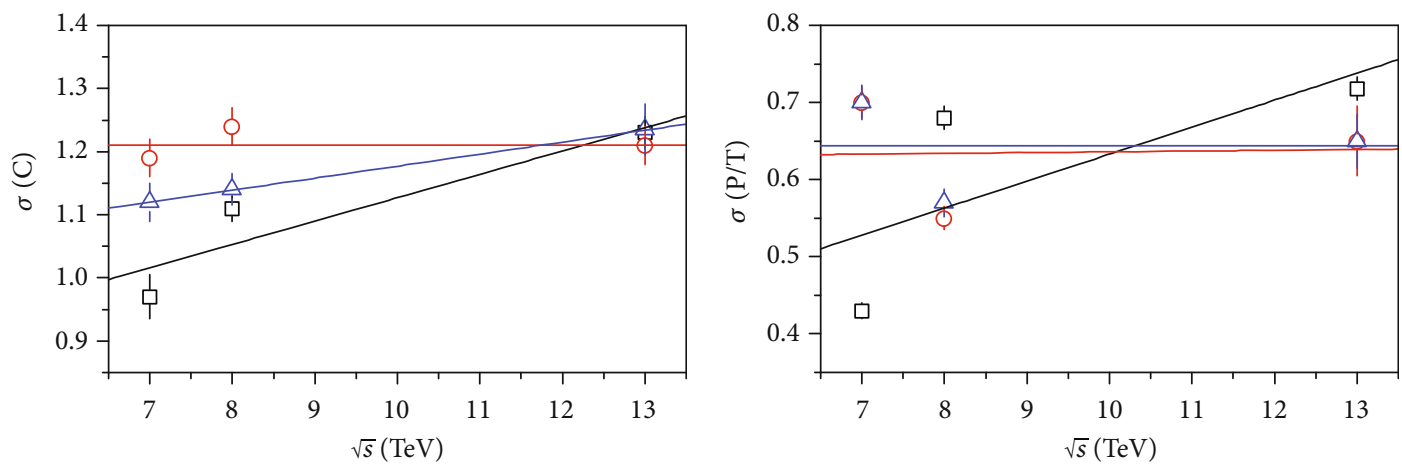

$\square t$
$\circ b$
$\triangle l$

$t$

$\circ b$

$\triangle l$

(c)

(d)

FIGURE 17: The relationship of the free parameter and collision energy. 
TABLE 4: Values of intercepts, slopes, and $\chi^{2} /$ dof corresponding to the lines in Figure 17.

\begin{tabular}{lccc}
\hline Particle & Intercept & Slope & $\chi^{2} /$ dof \\
\hline$T-\sqrt{s}$ & & & \\
$t$ & $21.068 \pm 1.276$ & $0.638 \pm 0.132$ & 0.113 \\
$b$ & $26.846 \pm 3.547$ & $0.066 \pm 0.366$ & 0.796 \\
$l$ & $6.835 \pm 1.149$ & $0.135 \pm 0.118$ & 1.643 \\
\hline$n-\sqrt{s}$ & & & \\
$t$ & $9.380 \pm 0.588$ & $-0.015 \pm 0.061$ & 0.258 \\
$b$ & $25.702 \pm 3.888$ & $-0.848 \pm 0.401$ & 3.324 \\
$l$ & $3.886 \pm 1.025$ & $0.071 \pm 0.106$ & 1.996 \\
\hline$\sigma(C)-\sqrt{s}$ & & & \\
$t$ & $0.757 \pm 0.111$ & $0.037 \pm 0.011$ & 9.802 \\
$b$ & $1.210 \pm 0.054$ & $0.000 \pm 0.006$ & 1.405 \\
$l$ & $0.987 \pm 0.001$ & $0.019 \pm 0.000$ & 0.001 \\
\hline$\sigma(P / T)-\sqrt{s}$ & & & \\
$t$ & $0.283 \pm 0.232$ & $0.035 \pm 0.024$ & 158.376 \\
$b$ & $0.626 \pm 0.163$ & $0.001 \pm 0.017$ & 41.913 \\
$l$ & $0.644 \pm 0.140$ & $0.001 \pm 0.014$ & 22.677 \\
\hline
\end{tabular}

energy increased. The values of $n$ about $t$ and $l$ do not show obvious change, and the values of $n$ about $b$ decreased when the collision energy increased. Besides, we found that there is no linear relationship $\sigma(P / T)$ and $\sqrt{s}$, so the values of $\chi^{2} /$ dof for this relationship are large which are listed in Table 4.

\section{Conclusion}

We summarize here our main observations and conclusions.

(a) We use the Tsallis-Pareto-type function to describe the transverse momentum cross-section spectrum of $t \bar{t}$ different cross sections in pp collisions at 7,8 , and $13 \mathrm{TeV}$. The values of the effective temperature of the interacting system $(T)$ and the nonextensivity of the process $(n)$ parameters are extracted and listed in Table 1

(b) The rapidity cross sections of the spectrum of $t \bar{t}$ different cross sections in pp collisions at 7,8 , and $13 \mathrm{TeV}$ are analyzed by the three-source Landau hydrodynamic model. We have obtained the values of the contribution of central emission source $\left(k_{\mathrm{C}}\right)$ and the width of rapidity distribution $\left(\sigma_{C}, \sigma_{P}\right)$ and calculated the values of $\chi^{2} /$ dof. The values of related parameters and $\chi^{2} /$ dof are given in Table 2. In fact, because of the relationship $k_{T}+k_{C}+k_{P}=1, k_{T}=k_{P}$, and $\sigma_{T}=\sigma_{P}$ (for the symmetric collision), we can calculate the contributions of the target and projectile source and the width of rapidity distribution of the target source (c) We have plotted the relationship of free parameters and collision energy in Figure 17. For the particles of $t, l$, and $b, \sigma(C)$ shows an increasing trend with the collision energy increased. And $T$ shows a slight increase with the collision energy increased; this phenomenon may be affected by QGP

(d) As has been mentioned in Section 3, the initial temperature $\left(T_{\mathrm{i}}\right)$ can be described by $\sqrt{\left\langle p_{T}^{2}\right\rangle / 2}$ approximately. In order to understand the excitation degree of the interacting system, we calculated the values of $\left\langle p_{T}\right\rangle$ and $\sqrt{\left\langle p_{T}^{2}\right\rangle / 2}$ by the curves in Figures $1,3,5$, and 7 . We found that the values of $\left\langle p_{T}\right\rangle$ and $\sqrt{\left\langle p_{T}^{2}\right\rangle / 2}$ are large; this could mean the high excitation degree of the interacting system

\section{Data Availability}

The data used to support the findings of this study are included within the article.

\section{Ethical Approval}

The authors declare that they are in compliance with ethical standards regarding the content of this paper.

\section{Conflicts of Interest}

The authors declare that they have no conflicts of interest regarding the publication of this paper.

\section{Acknowledgments}

This work is supported by Scientific and Technological Innovation Programs of Higher Education Institutions in Shanxi under Grant No. 2019L0804, the National Natural Science Foundation of China under Grant No. 11847003, and the university student innovation and entrepreneurship training program of Taiyuan Normal University No. CXCY 2084. This work has been published at http://arxiv.org/abs/2007 .12445 .

\section{References}

[1] CDF Collaboration, "Evidence for top quark production in $\overline{\mathrm{p}} \mathrm{p}$ collisions at $\sqrt{s}=1.8 \mathrm{TeV}$," Physical Review Letters, vol. 73, p. 225, 1994.

[2] CDF Collaboration, "Observation of top quark production in $\bar{p} p$ collisions with the collider detector at Fermilab," Physical Review Letters, vol. 74, no. 14, pp. 2626-2631, 1995.

[3] D0 Collaboration, "Observation of the top quark," Physical Review Letters, vol. 74, no. 14, pp. 2632-2637, 1995.

[4] CMS Collaboration, "Measurement of charged pion, kaon, and proton production in proton-proton collisions at $\sqrt{ } \mathrm{s}=13 \mathrm{TeV}$," Physical Review D, vol. 96, no. 11, article 112003, 2017.

[5] X. W. He, H. R. Wei, and F. H. Liu, "Chemical potentials of light hadrons and quarks from yield ratios of negative to positive particles in high energy pp collisions," Journal of Physics G: Nuclear and Particle Physics, vol. 46, no. 2, article 025102, 2019. 
[6] L. N. Gao, F. H. Liu, Y. Sun, Z. Sun, and R. A. Lacey, "Excitation functions of parameters extracted from three-source (net)proton rapidity distributions in $\mathrm{Au}-\mathrm{Au}$ and $\mathrm{Pb}-\mathrm{Pb}$ collisions over an energy range from AGS to RHIC," The European Physical Journal A, vol. 53, no. 3, p. 61, 2017.

[7] CMS collaboration, "Measurements of $t \bar{t}$ differential cross sections in proton-proton collisions at $\sqrt{ } \mathrm{s}=13 \mathrm{TeV}$ using events containing two leptons," Journal of High Energy Physics, vol. 2019, no. 2, p. 149, 2019.

[8] F. H. Liu, Y. Q. Gao, T. Tian, and B.-C. Li, "Unified description of transverse momentum spectrums contributed by soft and hard processes in high-energy nuclear collisions," The European Physical Journal A, vol. 50, no. 6, p. 94, 2014.

[9] L. N. Gao and F. H. Liu, "Comparing Erlang distribution and Schwinger mechanism on transverse momentum spectra in high energy collisions," Advances in High Energy Physics, vol. 2016, Article ID 1505823, 15 pages, 2016.

[10] L. N. Gao, F. H. Liu, and R. A. Lacey, "Excitation functions of parameters in Erlang distribution, Schwinger mechanism, and Tsallis statistics in RHIC BES program," The European Physical Journal A, vol. 52, no. 5, p. 137, 2016.

[11] UA1 Collaboration, "Transverse momentum spectra for charged particles at the CERN proton-antiproton collider," Physics Letters B, vol. 118, no. 1-3, pp. 167-172, 1982.

[12] R. Odorico, "Does a transverse energy trigger actually trigger on large-PT jets?," Physics Letters B, vol. 118, no. 1-3, pp. 151-154, 1982.

[13] T. Mizoguchi, M. Biyajima, and N. Suzuki, "Analyses of whole transverse momentum distributions in $\mathrm{p} \overline{\mathrm{p}}$ and $\mathrm{pp}$ collisions by using a modified version of Hagedorn's formula," International Journal of Modern Physics A: Particles and Fields; Gravitation; Cosmology; Nuclear Physics, vol. 32, no. 11, article 1750057, 2017.

[14] L. N. Gao, F. H. Liu, and B. C. Li, "Rapidity dependent transverse momentum spectra of heavy quarkonia produced in small collision systems at the LHC," Advances in High Energy Physics, vol. 2019, Article ID 6739315, 17 pages, 2019.

[15] J. Schwinger, "On gauge invariance and vacuum polarization," Physics Review, vol. 82, no. 5, pp. 664-679, 1951.

[16] R. C. Wang and C. Y. Wong, "Finite-size effect in the Schwinger particle-production mechanism," Physical Review D, vol. 38, no. 1, pp. 348-359, 1988.

[17] C. Tsallis, "Possible generalization of Boltzmann-Gibbs statistics," Journal of Statistical Physics, vol. 52, no. 1-2, pp. 479487, 1988.

[18] W. M. Alberico and A. Lavagno, "Non-extensive statistical effects in high-energy collisions," The European Physical Journal A, vol. 40, no. 3, p. 313, 2009.

[19] G. Wilk and Z. Wlodarezyk, "Multiplicity fluctuations due to the temperature fluctuations in high-energy nuclear collisions," Physical Review C, vol. 79, no. 5, article 054903, 2009.

[20] J. Cleymans, G. I. Lykasov, A. S. Parvan, A. S. Sorin, O. V. Teryaev, and D. Worku, "Systematic properties of the Tsallis distribution: energy dependence of parameters in high energy $p-p$ collisions," Physics Letters B, vol. 723, no. 4-5, pp. 351-354, 2013.

[21] J. Cleymans and D. Worku, "Relativistic thermodynamics: transverse momentum distributions in high-energy physics," The European Physical Journal A, vol. 48, no. 11, p. 160, 2012.

[22] C. Y. Wong and G. Wilk, "Tsallis fits to $p_{T}$ spectra and multiple hard scattering in $p p$ collisions at the LHC," Physical Review D, vol. 87, no. 11, article 114007, 2013.
[23] P. Carruthers and M. Duong-van, "New scaling law based on the hydrodynamical model of particle production," Physics Letters B, vol. 41, no. 5, pp. 597-601, 1972.

[24] P. Carruthers and M. Duong-van, "Rapidity and angular distributions of charged secondaries according to the hydrodynamical model of particle production," Physical Review D, vol. 8, no. 3, pp. 859-874, 1973.

[25] C. Y. Wong, "Landau hydrodynamics reexamined," Physical Review C, vol. 78, no. 5, article 054902, 2008.

[26] The CMS Collaboration, "Object definitions for top quark analyses at the particle level," Technical Report No. CMSNOTE-2017-004. CERN-CMS-NOTE-2017-004, CERN, Geneva, 2017.

[27] L. J. Gutay, A. S. Hirsch, R. P. Scharenberg, B. K. Srivastava, and C. Pajares, "De-confinement in small systems: clustering of color sources in high multiplicity $\bar{p} p$ collisions at $\mathrm{s}=$ 1.8TeV," International Journal of Modern Physics E: Nuclear Physics, vol. 24, no. 12, article 1550101, 2015.

[28] A. S. Hirsch, C. Pajares, R. P. Scharenberg, and B. K. Srivastava, "De-confinement in high multiplicity proton-proton collisions at LHC energies," 2018, https://arxiv.org/abs/1803 .02301 .

[29] P. Sahoo, S. De, S. K. Tiwari, and R. Sahoo, "Energy and centrality dependent study of deconfinement phase transition in a color string percolation approach at RHIC energies," The European Physical Journal A, vol. 54, article 136, 2018.

[30] The CMS Collaboration, "Measurement of differential topquark-pair production cross sections in $p p$ collisions at $\sqrt{ } \mathrm{s}=$ 7 TeV," European Physical Journal C, vol. 73, no. 3, article 2339, 2013.

[31] The CMS Collaboration, "Measurement of the differential cross section for top quark pair production in pp collisions at $\sqrt{ } \mathrm{s}=8 \mathrm{TeV}$," European Physical Journal C, vol. 75, no. 11, p. $542,2015$.

[32] H. R. Wei and F. H. Liu, "A study of transverse momentum distributions of jets produced in p-p,p-p-,d-Au, $\mathrm{Au}-\mathrm{Au}$, and $\mathrm{Pb}-\mathrm{Pb}$ collisions at high energies," Advances in High Energy Physics, vol. 2015, Article ID 263135, 17 pages, 2015. 